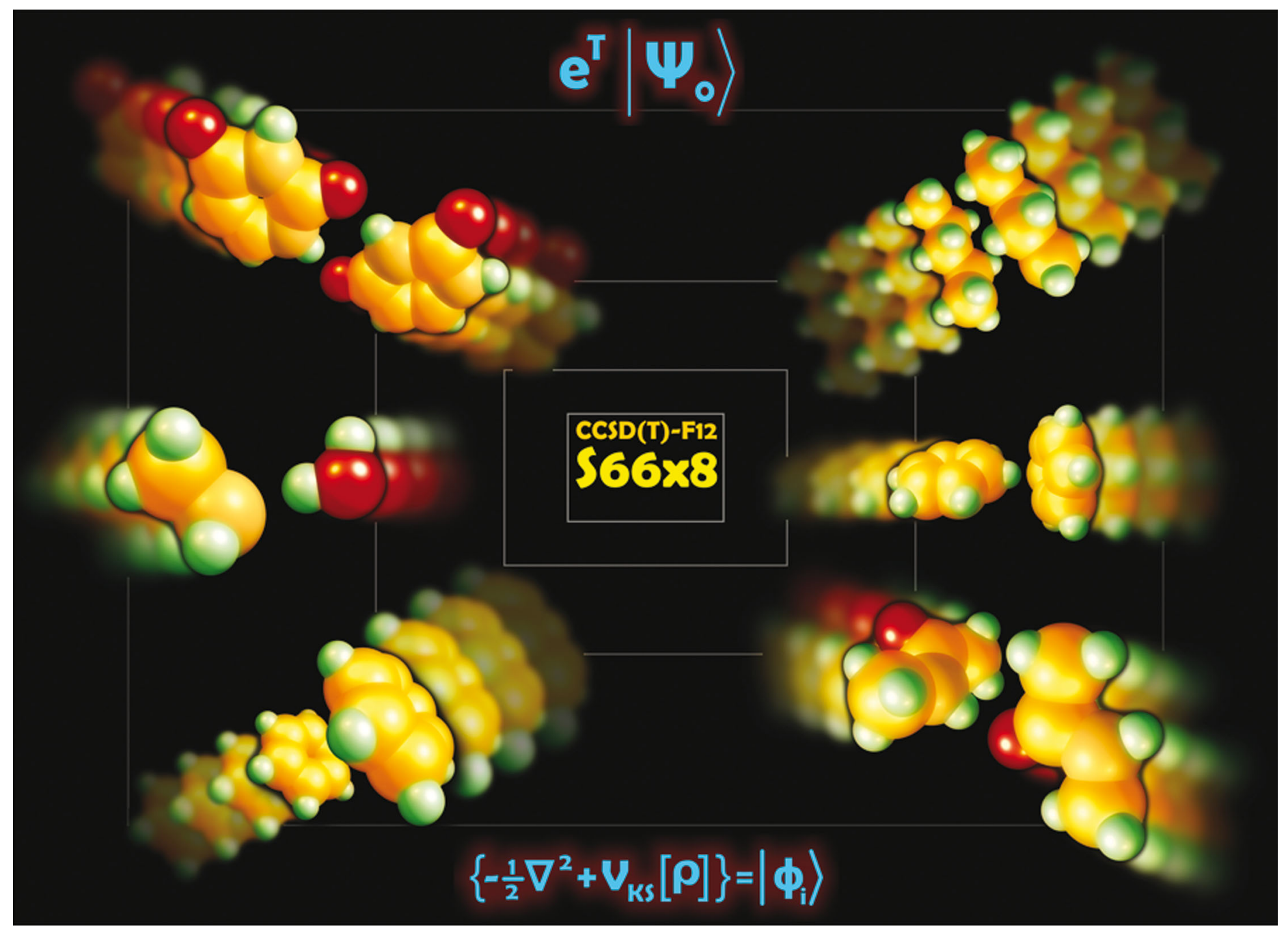

Showcasing research from the laboratory of Professor Jan Martin at the Department of Organic Chemistry, Weizmann Institute of Science, Rehovot, Israel

Title: The S66x 8 benchmark for noncovalent interactions revisited: explicitly correlated ab initio methods and density functional theory

The Martin group at Weizmann combines rigorous wavefunction ab initio methods (such as the Weizmann-n or Wn family) with development, validation, and application of density functional approaches. Their chemical interests focus on organometallic chemistry and catalysis, but recently are branching out in the direction of biomolecules and noncovalent interactions.

Research in the Kozuch group at Ben-Gurion University focuses on catalysis, tunneling effects on kinetics, and the nature of the chemical bond.

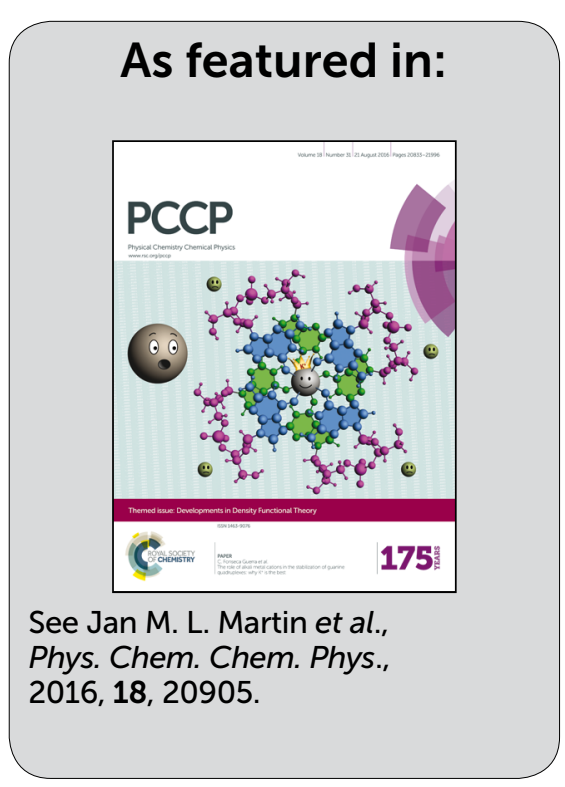

www.rsc.org/pccp 


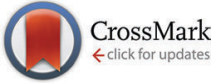

Cite this: Phys. Chem. Chem. Phys., 2016, 18, 20905

Received 30th January 2016, Accepted 1st March 2016

DOI: $10.1039 / c 6 c p 00688 d$

www.rsc.org/pccp

\title{
The S66x8 benchmark for noncovalent interactions revisited: explicitly correlated $a b$ initio methods and density functional theory $\dagger$
}

\author{
Brina Brauer $\ddagger^{a}$ Manoj K. Kesharwani $\ddagger^{a}$ Sebastian Kozuch ${ }^{b}$ and Jan M. L. Martin*a
}

The S66x8 dataset for noncovalent interactions of biochemical relevance has been re-examined by means of MP2-F12 and $\operatorname{CCSD}\left(F 12^{*}\right)(T)$ methods. We deem our revised benchmark data to be reliable to about $0.05 \mathrm{kcal} \mathrm{mol}^{-1} \mathrm{RMS}$. Most levels of DFT perform quite poorly in the absence of dispersion corrections: somewhat surprisingly, that is even the case for the double hybrids and for dRPA75. Analysis of optimized D3BJ parameters reveals that the main benefit of dRPA75 and DSD double hybrids alike is the treatment of midrange dispersion. dRPA75-D3BJ is the best performer overall at RMSD $=0.10 \mathrm{kcal} \mathrm{mol}^{-1}$. The nonlocal VV10 dispersion functional is especially beneficial for the double hybrids, particularly in DSD-PBEP86-NL (RMSD $=0.12 \mathrm{kcal} \mathrm{mol}^{-1}$ ). Other recommended dispersion-corrected functionals with favorable price/performance ratios are $\omega B 97 X-V$, and, surprisingly, B3LYP-D3BJ and BLYP-D3BJ (RMSDs of $0.23,0.20$ and $0.23 \mathrm{kcal} \mathrm{mol}^{-1}$, respectively). Without dispersion correction (but parametrized for midrange interactions) M06-2X has the lead (RMSD $=0.45 \mathrm{kcal} \mathrm{mol}^{-1}$ ). A collection of three energybased diagnostics yields similar information to an SAPT analysis about the nature of the noncovalent interaction. Two of those are the percentages of Hartree-Fock and of post-MP2 correlation effects in the interaction energy; the third, $\mathrm{CSPI}=\left[\mathrm{I} \mathrm{E}_{\mathrm{SS}}^{(2)}-\mathrm{IE}_{\mathrm{ab}}^{(2)}\right] /\left[\mathrm{I} \mathrm{E}_{\mathrm{SS}}^{(2)}+\mathrm{IE}_{\mathrm{ab}}^{(2)}\right]$ or its derived quantity $\mathrm{DEBC}=\mathrm{CSPI} /$ $\left(1+\mathrm{CSPI}^{2}\right)^{1 / 2}$, describes the character of the MP2 correlation contribution, ranging from 0 (purely dispersion) to 1 (purely other effects). In addition, we propose an improved, parameter-free scaling for the $(T)$ contribution based on the $E_{\mathrm{corr}}[\mathrm{CCSD}-\mathrm{F} 12 \mathrm{~b}] / E_{\mathrm{corr}}[\mathrm{CCSD}]$ and $E_{\mathrm{corr}}[\mathrm{CCSD}(\mathrm{F} 12 *)] / E_{\mathrm{corr}}[\mathrm{CCSD}]$ ratios. For Hartree-Fock and conventional DFT calculations, full counterpoise generally yields the fastest basis set convergence, while for double hybrids, half-counterpoise yields faster convergence, as previously established for correlated ab initio methods.

\section{Introduction}

Noncovalent interactions have great importance in many areas of research, particularly in chemistry and biological science. ${ }^{1,2}$ In biomolecules, noncovalent interactions play a major role in determining their structure and reactivity: hydrogen bonding, $\pi$ stacking, and dispersion interactions are among the most important noncovalent interactions.

All present-day computational methods capable of handling biomolecules with thousands of atoms, such as molecular

\footnotetext{
${ }^{a}$ Department of Organic Chemistry, Weizmann Institute of Science, 76100 Rehovot, Israel.E-mail: gershom@weizmann.ac.il; Fax: +97289343029

${ }^{b}$ Department of Chemistry, Ben-Gurion University of the Negev, 84105 Beer-Sheva, Israel

$\dagger$ Electronic supplementary information (ESI) available: Spreadsheet with revised reference interaction energies for the $566 \times 8$ dataset, interaction energies at various levels of theory and SAPT results; Cartesian coordinates (66 structures, XMol .xyz format) for the interpolated minimum geometries. See DOI: $10.1039 / \mathrm{c} 6 \mathrm{cp} 00688 \mathrm{~d}$ ‡ Equally contributing first authors.
}

mechanics force fields (e.g. ref. 3) and semiempirical methods (e.g., ref. 4 and 5), are highly approximated and heavily parametrized. Ideally, parametrization of such approximate methods would be based on experimental observations; in practice, this is an intractable problem as experimental data are not available in sufficient quantity or in isolation from various environmental or dynamical effects that cannot easily be included in the approximate method during the many evaluation cycles required for parametrization. Highly accurate $a b$ initio computational data represent a convenient alternative.

From a SAPT (symmetry-adapted [intermolecular] perturbation theory) perspective (see ref. 6-8 for recent reviews), the interaction energy of a noncovalent dimer can be decomposed into four main components: exchange repulsion $\left(E_{\text {exc }}\right)$, electrostatic attraction $\left(E_{\text {elst }}\right)$, induced electrostatic interactions $\left(E_{\text {ind }}\right)$, and dispersion forces $\left(E_{\text {disp }}\right)$. The balance of their relative importance changes between different types of systems, ${ }^{9}$ as well as with distance, but dispersion (which is a long-range electron correlation effect) matters in all of them: indeed, in certain systems (such as noble 
gas dimers or alkane dimers) it is the glue that holds the dimer together at all. Electron correlation however also contributes in higher order to induced and electrostatic forces, particularly at shorter distances. To sum up, no accurate treatment of long-range correlation effects is possible without accounting for electron correlation. $^{10}$

Coupled-cluster correlated methods with sufficiently large basis sets are known to accurately reproduce these interactions, but their high computational cost and massive resource demands limit their use to small benchmark systems.

Such benchmark data, for a representative set of small systems, do enable the validation and/or calibration of less demanding, more approximate methods, such as density functional theory.

In the past decade, a number of databases have been proposed for noncovalent interactions. An early one that has been used in the parametrization of a number of empirical density functionals is $\mathrm{S} 22,{ }^{11}$ which are 22 noncovalent complexes ranging from water and methane dimers to the adenine-thymine base pair (both Watson-Crick and stacked). Its $a b$ initio reference data were recently comprehensively revised. ${ }^{12}$

In order to have a broader set that is more representative of interactions one might see in biomolecules, Hobza and coworkers assembled a larger S66 set ${ }^{9,13}$ of 66 noncovalent pairs, generated from 14 monomers in various combinations. The selection of monomers was based on their frequency as motifs or functional groups in the most commonly found biomolecules. The S66 set was designed with a balance in mind between electrostatic dominated (hydrogen bonding), dispersion dominated (including $\pi$ stacking), and mixed-influenced complexes. Single hydrogen bonds, aromatic-aliphatic, and aliphatic-aliphatic interactions are also incorporated into the S66 set, which were not adequately covered by the narrower S22 dataset.

In an actual biomolecule, such interactions would not necessarily occur near the equilibrium inter-monomer separation, but at the separation dictated by the geometry of the system (e.g., by the secondary structure of the protein). Hence, the Hobza group extended the S66 set by considering each dimer at eight different inter-monomer separations: $0.90 r_{\mathrm{e}}, 0.95 r_{\mathrm{e}}, r_{\mathrm{e}}, 1.05 r_{\mathrm{e}}, 1.10 r_{\mathrm{e}}, 1.25 r_{\mathrm{e}}$, $1.5 r_{\mathrm{e}}$, and $2 r_{\mathrm{e}}$, where $r_{\mathrm{e}}$ is the equilibrium distance (the monomers were separated out without further geometry optimization). Thus, the S66x8 dataset was created, which is the subject of the present investigation. A full listing of the systems, together with the final recommended values obtained in the present work, is presented in Table 1. Reference geometries were taken "as is" from the Benchmark Energy and Geometry Database (www.begdb.com). ${ }^{14}$

Several studies have been published regarding the performance of lower $a b$ initio, DFT and double-hybrid DFT methods using S66 and/or S66x8 as a benchmark. ${ }^{15-20}$ Those results use the originally reported $\operatorname{CCSD}(\mathrm{T}) / \mathrm{CBS}$ calculated interaction energies as the reference. Those were based on extrapolated MP2 limits combined with additive "high-level corrections" (HLCs) - that is, CCSD(T)-MP2 differences - in the meager augcc-pVDZ basis set. Hobza and coworkers ${ }^{13}$ re-evaluated the HLCs for just S66 (which is almost, but not quite, equivalent to the 1.0 $r_{\mathrm{e}}$ 'slice' of S66x8): the RMSD (root mean square difference) between the original ${ }^{9}$ and revised ${ }^{13}$ sets is $0.10 \mathrm{kcal} \mathrm{mol}^{-1}$, with the individual largest positive difference of $0.32 \mathrm{kcal} \mathrm{mol}^{-1}$ for acetic acid dimer, and largest negative difference of $0.12 \mathrm{kcal} \mathrm{mol}^{-1}$ for benzene-uracil. While this may not sound like a great deal, we shall show below that this is comparable with the accuracy of the best DFT methods available nowadays.

In reference to originally reported S66 and S66x8 database, Grimme and coworkers ${ }^{17}$ have assessed the performance of several DFT methods, also have tested their own developed dispersion-correction schemes DFT-D3 and DFT-D3BJ. Aragó et al. ${ }^{15}$ and $\mathrm{Yu}^{16}$ have considered the S66 database to evaluate the efficacy of nonlocal van der Waals corrections for the doublehybrid DFT and spin-component-scaled double-hybrid DFT methods, respectively. The Hobza group ${ }^{18}$ themselves have evaluated the performance of the MP2 variants for noncovalent interactions of the S66 benchmark set.

Basis set convergence of orbital-based $\operatorname{CCSD}(\mathrm{T})$ is quite slow, debilitatingly so in fact, for our purposes. Explicitly correlated ${ }^{21,22}$ (in practice nowadays, F12) ${ }^{23}$ approaches offer succor here: for many applications, we can expect a gain of 2-3 angular momentum steps. ${ }^{24-26}$ Furthermore, ${ }^{26,27}$ it has been reported that the combination of cc-pVDZ-F12 HLCs (high-level corrections, defined as the CCSD(T)-F12-MP2-F12 difference) with larger-basis MP2-F12 energetics yields excellent results for noncovalent interactions.

In this paper, we are reporting a revision of the reference interaction energies for the S66x8 dataset by means of explicitly correlated MP2 and coupled cluster methods. These interaction energy data will then be used to evaluate the performance of various wavefunction $a b$ initio and density functional, as well as double-hybrid density functionals, which are fifth-rung DFT functionals from one perspective and occupy the twilight zone between wavefunction and DFT methods from another. In most cases, performance of DFT methods for noncovalent interactions is very poor unless dispersion corrections are included: both molecular mechanics-like corrections and nonlocal dispersion functionals will be considered in this work. The issue of BSSE (basis set superposition error) for explicitly correlated methods was considered in an earlier study ${ }^{26}$ and will be re-examined here for all methods.

\section{Computational details}

All calculations were performed on the Faculty of Chemistry cluster at the Weizmann Institute of Science. Most wavefunctionbased $a b$ initio calculations were carried out using MOLPRO 2012.1, ${ }^{28}$ while Turbomole ${ }^{29} 6.6$ was employed for some MP3-F12 calculations. The density functional calculations were performed using either the Gaussian 09 Rev. D.01 package, ${ }^{30}$ or primarily for the double hybrids a locally modified version of ORCA. ${ }^{31}$ The latter was primarily used for the double hybrids, owing to the availability of the RI-MP2 (resolution of the identity MP2) method, ${ }^{32,33}$ approximation for the MP2-like step.

For conventional, orbital-based, $a b$ initio calculations we mostly employed correlation-consistent ${ }^{34-37}$ basis sets. In general, we combined diffuse-function augmented sets aug-cc-pVnZ 
Table 1 Systems in the $566 \times 8$ dataset and final recommended dissociation energies $\left(\mathrm{kcal} \mathrm{mol}^{-1}\right)$ obtained in the present work

\begin{tabular}{|c|c|c|c|c|c|c|c|c|}
\hline & $0.90 r_{\mathrm{e}}$ & $0.95 r_{\mathrm{e}}$ & $1.00 r_{\mathrm{e}}$ & $1.05 r_{\mathrm{e}}$ & $1.10 r_{\mathrm{e}}$ & $1.25 r_{\mathrm{e}}$ & $1.50 r_{\mathrm{e}}$ & $2.00 r_{\mathrm{e}}$ \\
\hline \multicolumn{9}{|l|}{ Hydrogen bonding } \\
\hline 01 water $\cdots$ water & 4.659 & 4.954 & 4.951 & 4.768 & 4.487 & 3.473 & 2.113 & 0.872 \\
\hline 02 water $\cdots \mathrm{MeOH}$ & 5.293 & 5.630 & 5.634 & 5.434 & 5.119 & 3.967 & 2.394 & 0.955 \\
\hline 03 water $\cdots \mathrm{MeNH}_{2}$ & 6.577 & 6.937 & 6.929 & 6.689 & 6.315 & 4.932 & 2.982 & 1.143 \\
\hline 04 water $\cdots$ peptide & 7.748 & 8.149 & 8.153 & 7.907 & 7.514 & 6.023 & 3.844 & 1.442 \\
\hline $05 \mathrm{MeOH} \cdots \mathrm{MeOH}$ & 5.396 & 5.787 & 5.824 & 5.644 & 5.337 & 4.172 & 2.538 & 1.015 \\
\hline $06 \mathrm{MeOH} \cdots \mathrm{MeNH}_{2}$ & 7.067 & 7.550 & 7.603 & 7.385 & 7.005 & 5.517 & 3.352 & 1.277 \\
\hline $07 \mathrm{MeOH} \cdots$ peptide & 7.784 & 8.270 & 8.330 & 8.116 & 7.737 & 6.228 & 3.668 & 1.104 \\
\hline $08 \mathrm{MeOH} \cdots$ water & 4.684 & 5.032 & 5.063 & 4.901 & 4.629 & 3.611 & 2.208 & 0.909 \\
\hline $09 \mathrm{MeNH}_{2} \cdots \mathrm{MeOH}$ & 2.862 & 3.078 & 3.063 & 2.917 & 2.704 & 1.984 & 1.104 & 0.396 \\
\hline $10 \mathrm{MeNH}_{2} \cdots \mathrm{MeNH}_{2}$ & 3.724 & 4.113 & 4.165 & 4.016 & 3.756 & 2.793 & 1.309 & 0.390 \\
\hline $11 \mathrm{MeNH}_{2} \cdots$ peptide & 5.009 & 5.409 & 5.453 & 5.277 & 4.976 & 3.230 & 1.413 & 0.460 \\
\hline $12 \mathrm{MeNH}_{2} \cdots$ water & 6.834 & 7.272 & 7.300 & 7.070 & 6.688 & 5.234 & 3.158 & 1.199 \\
\hline 13 peptide $\cdots \mathrm{MeOH}$ & 5.811 & 6.224 & 6.278 & 6.109 & 5.810 & 4.646 & 2.970 & 1.315 \\
\hline 14 peptide $\cdots \mathrm{MeNH}_{2}$ & 6.938 & 7.446 & 7.536 & 7.360 & 7.021 & 5.639 & 3.567 & 1.498 \\
\hline 15 peptide $\cdots$ peptide & 8.204 & 8.696 & 8.767 & 8.569 & 8.204 & 6.728 & 4.457 & 1.793 \\
\hline 16 peptide $\cdots$ water & 4.801 & 5.150 & 5.191 & 5.044 & 4.790 & 3.825 & 2.468 & 1.139 \\
\hline 17 uracil $\cdots$ uracil (BP) & 16.229 & 17.358 & 17.583 & 17.214 & 16.473 & 13.350 & 8.462 & 3.380 \\
\hline 18 water $\cdots$ pyridine & 6.527 & 6.918 & 6.928 & 6.701 & 6.336 & 4.965 & 3.026 & 1.195 \\
\hline $19 \mathrm{MeOH} \cdots$ pyridine & 6.944 & 7.445 & 7.520 & 7.324 & 6.963 & 5.525 & 3.403 & 1.343 \\
\hline $20 \mathrm{AcOH} \cdots \mathrm{AcOH}$ & 17.970 & 19.228 & 19.469 & 19.049 & 18.219 & 14.736 & 9.289 & 3.611 \\
\hline $21 \mathrm{AcNH}_{2} \cdots \mathrm{AcNH}_{2}$ & 15.335 & 16.375 & 16.559 & 16.188 & 15.476 & 12.544 & 8.054 & 3.020 \\
\hline $22 \mathrm{AcOH} \cdots$ uracil & 18.421 & 19.632 & 19.877 & 19.488 & 18.697 & 15.325 & 9.953 & 4.179 \\
\hline $23 \mathrm{AcNH}_{2} \cdots$ uracil & 18.182 & 19.310 & 19.557 & 19.217 & 18.501 & 15.386 & 10.320 & 4.691 \\
\hline \multicolumn{9}{|l|}{$\pi$ stacking } \\
\hline 24 benzene $\cdots$ benzene $(\pi-\pi)$ & 0.105 & 2.016 & 2.725 & 2.813 & 2.607 & 1.578 & 0.515 & 0.072 \\
\hline 25 pyridine $\cdots$ pyridine $(\pi-\pi)$ & 1.201 & 3.111 & 3.809 & 3.865 & 3.607 & 2.396 & 0.990 & 0.246 \\
\hline 26 uracil $\cdots$ uracil $(\pi-\pi)$ & 7.976 & 9.640 & 9.976 & 9.590 & 8.848 & 6.217 & 3.196 & 1.034 \\
\hline 27 benzene $\cdots$ pyridine $(\pi-\pi)$ & 0.602 & 2.636 & 3.358 & 3.414 & 3.159 & 2.012 & 0.752 & 0.155 \\
\hline 28 benzene $\cdots$ uracil $(\pi-\pi)$ & 3.492 & 5.222 & 5.741 & 5.604 & 5.144 & 3.363 & 1.412 & 0.271 \\
\hline 29 pyridine $\cdots$ uracil $(\pi-\pi)$ & 3.679 & 6.170 & 6.847 & 6.637 & 6.039 & 3.937 & 1.824 & 0.552 \\
\hline 30 benzene . ethene & 0.111 & 1.027 & 1.350 & 1.366 & 1.237 & 0.692 & 0.183 & 0.006 \\
\hline 31 uracil . ethene & 2.519 & 3.209 & 3.365 & 3.235 & 2.966 & 2.009 & 0.951 & 0.262 \\
\hline 32 uracil ...ethyne & 2.708 & 3.507 & 3.702 & 3.571 & 3.281 & 2.232 & 1.054 & 0.278 \\
\hline 33 pyridine $\cdots$ ethene & 0.765 & 1.541 & 1.808 & 1.799 & 1.651 & 1.030 & 0.374 & 0.049 \\
\hline \multicolumn{9}{|l|}{ London dispersion complexes } \\
\hline 34 pentane $\cdots$ pentane & 2.919 & 3.674 & 3.820 & 3.651 & 3.337 & 2.257 & 1.066 & 0.278 \\
\hline 35 neopentane $\cdots$ pentane & 1.913 & 2.542 & 2.651 & 2.518 & 2.282 & 1.516 & 0.711 & 0.191 \\
\hline 36 neopentane $\cdots$ neopentane & 1.484 & 1.758 & 1.790 & 1.698 & 1.551 & 1.060 & 0.512 & 0.139 \\
\hline 37 cyclopentane $\cdots$ neopentane & 1.669 & 2.299 & 2.443 & 2.354 & 2.161 & 1.482 & 0.716 & 0.194 \\
\hline 38 cyclopentane $\cdots$ cyclopentane & 2.301 & 2.894 & 3.038 & 2.893 & 2.621 & 1.731 & 0.804 & 0.211 \\
\hline 39 benzene $\cdots$ cyclopentane & 2.143 & 3.243 & 3.584 & 3.492 & 3.193 & 2.094 & 0.918 & 0.199 \\
\hline 40 benzene $\cdots$ neopentane & 1.859 & 2.683 & 2.909 & 2.824 & 2.591 & 1.729 & 0.786 & 0.196 \\
\hline 41 uracil $\cdots$ pentane & 3.932 & 4.792 & 4.934 & 4.689 & 4.163 & 2.508 & 1.012 & 0.228 \\
\hline 42 uracil . . cyclopentane & 3.146 & 4.062 & 4.221 & 4.001 & 3.615 & 2.352 & 1.052 & 0.263 \\
\hline 43 uracil $\cdots$ neopentane & 2.954 & 3.684 & 3.782 & 3.566 & 3.217 & 2.102 & 0.955 & 0.244 \\
\hline 44 ethene $\cdots$ pentane & 1.647 & 1.966 & 1.987 & 1.861 & 1.674 & 1.093 & 0.497 & 0.123 \\
\hline 45 ethyne $\cdots$ pentane & 1.045 & 1.563 & 1.698 & 1.638 & 1.490 & 0.966 & 0.421 & 0.098 \\
\hline 46 peptide $\cdots$ pentane & 3.814 & 4.264 & 4.298 & 4.096 & 3.775 & 2.662 & 1.212 & 0.298 \\
\hline \multicolumn{9}{|l|}{ Mixed influence complexes } \\
\hline 47 benzene $\cdots$ benzene (TS) & 1.657 & 2.603 & 2.898 & 2.853 & 2.643 & 1.804 & 0.856 & 0.237 \\
\hline 48 pyridine $\cdots$ pyridine (TS) & 2.547 & 3.336 & 3.566 & 3.489 & 3.255 & 2.328 & 1.206 & 0.384 \\
\hline 49 benzene $\cdots$ pyridine (TS) & 2.099 & 3.064 & 3.354 & 3.291 & 3.056 & 2.134 & 1.075 & 0.345 \\
\hline 50 benzene $\cdots$ ethyne $(\mathrm{CH}-\pi)$ & 1.847 & 2.636 & 2.865 & 2.800 & 2.593 & 1.802 & 0.903 & 0.277 \\
\hline 51 ethyne $\cdots$ ethyne (TS) & 1.203 & 1.464 & 1.516 & 1.456 & 1.341 & 0.934 & 0.463 & 0.135 \\
\hline 52 benzene $\cdots$ AcOH $(\mathrm{OH}-\pi)$ & 4.002 & 4.613 & 4.737 & 4.583 & 4.283 & 3.156 & 1.722 & 0.564 \\
\hline 53 benzene $\cdots \mathrm{AcNH}_{2}(\mathrm{NH}-\pi)$ & 3.849 & 4.320 & 4.408 & 4.270 & 4.008 & 3.005 & 1.668 & 0.492 \\
\hline 54 benzene $\cdots$ water $(\mathrm{OH}-\pi)$ & 2.766 & 3.186 & 3.250 & 3.121 & 2.896 & 2.105 & 1.160 & 0.419 \\
\hline 55 benzene $\cdots \mathrm{MeOH}(\mathrm{OH}-\pi)$ & 3.403 & 4.014 & 4.168 & 4.057 & 3.804 & 2.813 & 1.542 & 0.526 \\
\hline 56 benzene $\cdots \mathrm{MeNH}_{2}(\mathrm{NH}-\pi)$ & 2.457 & 3.051 & 3.209 & 3.109 & 2.861 & 1.960 & 0.951 & 0.267 \\
\hline 57 benzene $\cdots$ peptide $(\mathrm{NH}-\pi)$ & 3.756 & 4.978 & 5.313 & 5.183 & 4.825 & 3.458 & 1.837 & 0.632 \\
\hline 58 pyridine $\cdots$ pyridine $(\mathrm{CH}-\mathrm{N})$ & 2.989 & 3.970 & 4.259 & 3.973 & 3.517 & 2.226 & 1.041 & 0.287 \\
\hline 59 ethyne $\cdots$ water $(\mathrm{CH}-\mathrm{O})$ & 2.608 & 2.873 & 2.907 & 2.807 & 2.634 & 2.003 & 1.182 & 0.461 \\
\hline 60 ethyne $\cdots$ AcOH $(\mathrm{OH}-\pi)$ & 4.364 & 4.844 & 4.908 & 4.727 & 4.414 & 3.263 & 1.776 & 0.559 \\
\hline 61 pentane $\cdots$ AcOH & 2.741 & 2.938 & 2.913 & 2.761 & 2.544 & 1.812 & 0.802 & 0.177 \\
\hline 62 pentane $\cdots \mathrm{AcNH}_{2}$ & 3.193 & 3.538 & 3.540 & 3.353 & 3.072 & 2.140 & 1.061 & 0.283 \\
\hline 63 benzene $\cdots$ AcOH & 2.691 & 3.581 & 3.798 & 3.660 & 3.354 & 2.260 & 1.040 & 0.271 \\
\hline
\end{tabular}


Table 1 (continued)

\begin{tabular}{|c|c|c|c|c|c|c|c|c|}
\hline 65 pyridine . . ethyne & 3.701 & 4.048 & 4.104 & 3.980 & 3.755 & 2.888 & 1.694 & 0.627 \\
\hline
\end{tabular}

( $\mathrm{n}=\mathrm{D}, \mathrm{T}, \mathrm{Q}, 5)$ on nonhydrogen atoms with the underlying regular cc-pVnZ basis sets on hydrogen. This practice has been denoted variously in the literature as aug'-cc-pVnZ by Del Bene, ${ }^{38} \mathrm{~A}^{\prime} \mathrm{Vnz}$ by ourselves, hanz ("heavy-augmented") by the Hobza group, ${ }^{13}$ heavy-aug-cc-pVnz by the Sherrill group, ${ }^{39}$ and (with a calendric pun) jul-cc-pVnZ by Papajak \& Truhlar. ${ }^{40}$

For some of the $a b$ initio calculations, and all of the DFT calculations, we employed the Weigend-Ahlrichs basis sets ${ }^{41}$ def2-QZVP, def2-TZVPP, def2-TZVP and def2-SVP, as well as the diffuse-function augmented def2-QZVPD basis set. ${ }^{42}$ In ORCA, we employed the corresponding auxiliary basis sets ${ }^{43}$ for simultaneously fitting Coulomb and exchange, and the associated RI-MP2 auxiliary basis sets ${ }^{44}$ for the double-hybrid calculations in ORCA. The Weigend-Ahlrichs family seeks to strike a balance between the requirements of DFT and wavefunction $a b$ initio calculations, and was therefore deemed especially appropriate for the double hybrids.

Single-point explicitly correlated $\operatorname{CCSD}(\mathrm{T})\left(\mathrm{F} 12^{*}\right), \operatorname{CCSD}(\mathrm{T})-\mathrm{F} 12 \mathrm{~b}$ and RI-MP2-F12 calculations were carried out using the cc-pVnZF12 (where $\mathrm{n}=\mathrm{D}, \mathrm{T}, \mathrm{Q}$ ) basis sets of Peterson et al. ${ }^{45}$ in conjunction with the associated auxiliary and complementary auxiliary (CABS) basis sets. ${ }^{46,47}$ The cc-pVnZ-F12 family was specifically developed with explicitly correlated calculations.

For some calibration calculations, the even larger cc-pV5Z-F12 basis set ${ }^{27}$ was employed, which effectively corresponds to the basis set limit. Here, we employed a combination of Weigend's aug-cc-pV5Z/JKFIT basis set ${ }^{43}$ for the Coulomb and exchange elements and Hättig's aug-cc-pwCV5Z/MP2FIT basis set ${ }^{44}$ for both the RI-MP2 parts and for the CABS.

As recommended in ref. 48, the geminal exponents were set to $\beta=0.9$ for cc-pVDZ-F12 and $\beta=1.0$ for cc-pVTZ-F12 and cc-pVQZF12; for cc-pV5Z-F12 we used $\beta=1.2$ as specified in ref. 27 . The SCF component was improved through the "CABS correction". ${ }^{49,50}$

For the (T) term, which does not benefit from the F12, we considered three different corrections for basis set expansion: (a) the Marchetti-Werner approximation, ${ }^{51,52}$ denoted $\operatorname{CCSD}\left(\mathrm{T}^{*}\right)-\mathrm{F} 12 \mathrm{~b}$, in which the $(\mathrm{T})$ contribution is scaled by the $E_{\text {corr }}[\mathrm{MP} 2-\mathrm{F} 12] / E_{\text {corr }}[\mathrm{MP} 2]$ correlation energy ratio; (b) analogues, denoted $\operatorname{CCSD}(\mathrm{Tb})$ and $\operatorname{CCSD}(\mathrm{Tc})$, in which the respective $E_{\text {corr }}[\mathrm{CCSD}-\mathrm{F} 12 \mathrm{~b}] / E_{\text {corr }}[\mathrm{CCSD}]$ and $E_{\text {corr }}\left[\mathrm{CCSD}\left(\mathrm{F} 12^{*}\right)\right] / E_{\text {corr }}[\mathrm{CCSD}]$ ratios were substituted; and (c) uniform scaling of the (T) contributions, denoted CCSD(Ts)-F12b, ${ }^{27}$ in which the (T) contributions are multiplied by constant scaling factors of 1.1413 for cc-pVDZ-F12 and 1.0527 for cc-pVTZ-F12 (Table 3 in ref. 27). Options (a) and (b) are not strictly size-consistent, but can be rendered so by applying the dimer $E_{\text {corr }}[\mathrm{MP} 2-\mathrm{F} 12] / E_{\text {corr }}[\mathrm{MP} 2]$, $E_{\text {corr }}[\mathrm{CCSD}-\mathrm{F} 12 \mathrm{~b}] / E_{\text {corr }}[\mathrm{CCSD}], \quad$ viz. $\quad E_{\text {corr }}\left[\mathrm{CCSD}\left(\mathrm{F} 12^{*}\right)\right] / E_{\text {corr }}[\mathrm{CCSD}]$ ratios also to the monomers: this is indicated by the notation $\operatorname{CCSD}\left(\mathrm{T}^{*}{ }_{\mathrm{sc}}\right), \operatorname{CCSD}\left(\mathrm{Tb}_{\mathrm{sc}}\right)$, and $\operatorname{CCSD}\left(\mathrm{Tc}_{\mathrm{sc}}\right)$, respectively.
As byproducts of the MP2 and MP2-F12 calculations, we also obtain spin-component-scaled varieties such as SCS-MP2-F12, ${ }^{53-55}$ SCS(MI)MP2, and S2-MP2. ${ }^{56}$

The following DFT functionals were considered (grouped by rung on the Perdew ${ }^{57}$ "Jacob's Ladder"):

- on the second (GGA) rung, BP86, ${ }^{58,59} \mathrm{BLYP},{ }^{58,60}$ and PBE; ${ }^{61}$

- on the third (meta-GGA) rung, TPSS, ${ }^{62}$ and M06L $;^{63}$

- on the fourth (occupied-orbital dependent) rung, the hybrid ${ }^{64}$ functionals B3LYP, ${ }^{60,65,66}$ B3PW91, ${ }^{65,67}$ PBE0, ${ }^{68}$ TPSS0, ${ }^{69,70} \mathrm{APF},{ }^{71} \mathrm{M} 06,{ }^{63}$ and $\mathrm{M} 06-2 \mathrm{X} ;{ }^{63}$ as well as the rangeseparated hybrids M11, ${ }^{72}$ CAM-B3LYP, ${ }^{73}$ LC- $\omega$ PBE $^{74} \omega B$ B7X-D3, ${ }^{75}$ and $\omega \mathrm{B} 97 \mathrm{X}-\mathrm{V} .^{76}$

- and on the fifth (virtual-orbital dependent) rung, the double hybrids B2PLYP, ${ }^{77}$ B2GP-PLYP, ${ }^{78}$ and the spin component scaled double hybrids DSD-PBEP86, ${ }^{79}$ DSD-PBEPBE, ${ }^{80}$ and DSD-PBEB95 ${ }^{80}$ methods. The dRPA75 $\operatorname{method}^{81}$ represents approaches with correlation based on the random phase approximation (RPA). ${ }^{82}$

Further, we have also considered the following types of empirical dispersion corrections (for a review see ref. 83) for DFT energies:

(a) Grimme's 2006 version, denoted by the suffix “-D2" using Grimme's expression

$$
E_{\mathrm{disp}}=-S_{6} \sum_{i=1}^{N_{\mathrm{at}}-1} \sum_{j=i+1}^{N_{\mathrm{at}}} \frac{C_{6}^{i j}}{R_{i j}{ }^{6}} f_{\mathrm{dmp}}\left(R_{i j}\right)
$$

in which the damping function is taken as

$$
f_{\mathrm{dmp}}\left(R_{i j}\right)=\left[1+\exp \left(-\alpha\left(\frac{R_{i j}}{s_{\mathrm{R}} R_{\mathrm{r}}}-1\right)\right)\right]^{-1}
$$

where $s_{6}$ is a scaling factor that depends only of the functional used, $C_{6}^{i j} \approx\left(C_{6}^{i} C_{6}^{j}\right)^{1 / 2}$ is the dispersion coefficient for the atom pair $i j$ computed by using a geometric mean, $R_{\mathrm{r}}=R_{\mathrm{vdW}, i}+R_{\mathrm{vdW}, j}$ is the sum of the van der Waals radii of the two atoms in question, and specific numerical values for the atomic Lennard-Jones constants $C_{6}^{i}$ and the van der Waals radii have been taken from ref. 84. The length-scaling factor $s_{\mathrm{R}}=1.0$ and hysteresis exponent $\alpha=20.0$ were set as in ref. 85 .

(b) Grimme's DFT-D3 ${ }^{86,87}$ version with Becke-Johnson damping, denoted by the suffix "-D3BJ"

$$
E_{\mathrm{disp}}^{\mathrm{D} 3(\mathrm{BJ})}=-\sum_{i>j} s_{6} \frac{C_{6, i j}}{R_{i j}{ }^{6}+\left[f\left(R_{\mathrm{r}}\right)\right]^{6}}+s_{8} \frac{C_{8, i j}}{R_{i j}{ }^{8}+\left[f\left(R_{\mathrm{r}}\right)\right]^{8}}
$$

in which $f\left(R_{\mathrm{r}}\right)=a_{1} R_{\mathrm{r}}+a_{2}$. This modified cutoff function does not fade to zero at short distance but to a small finite value.

Where parameters were not available from the literature, or from Prof. Grimme's website, we have optimized them ourselves against our best S66x8 reference data, using an adaptation of the 
Table 2 Basis set extrapolation exponents for various energy components, rounded to four decimal places

\begin{tabular}{|c|c|c|c|c|}
\hline & $\{\mathrm{T}, \mathrm{Q}\}$ & $\{\mathrm{Q}, 5\}$ & $\{5,6\}$ & \\
\hline SCF alternate ${ }^{a}$ & 5.1507 & 10.3626 & 12.2568 & Schwenke ${ }^{94}$ \\
\hline MP2 & 2.5313 & 2.7399 & 2.8349 & Hill et al., ${ }^{48}$ Table 8 \\
\hline CCSD & 3.0840 & 3.2711 & 3.1937 & Schwenke $^{94}$ \\
\hline CCSD-MP2 & 2.0926 & 2.2459 & 2.3546 & Ranasinghe \& Petersson, ${ }^{95}$ Fig. 6 \\
\hline$(\mathrm{T})$ & 2.9988 & 3.6018 & 3.2279 & Schwenke $^{94}$ \\
\hline CCSD-F12b & 4.5960 & 6.0642 & N/A & $\{\mathrm{T}, \mathrm{Q}\}$ Hill et al.,${ }^{48}$ Table $10 ;\{\mathrm{Q}, 5\}$ this work \\
\hline (T) post F12 & 2.8950 & & & $\{\mathrm{~T}, \mathrm{Q}\}$ Hill et al., ${ }^{48}$ Table 11 \\
\hline
\end{tabular}

BOBYQA (Bound Optimization BY Quadratic Approximation) optimization program of Powell. ${ }^{88}$

In addition to these molecular mechanics-like corrections, we have also considered the Vydrov-van Voorhis (VV10) "nonlocal" (NL) dispersion functional, ${ }^{89}$ in which an a posteriori correction is obtained from the electron density. The required short-range attenuation parameter, $b$, used for various DFT-NL calculations were obtained from ref. 90 for the conventional DFT functionals and optimized in our group for the DSD double hybrids: the various values are listed in Table 1 of ref. 91 These calculations were carried out using its implementation in ORCA.

The values for DSD-PBEP86-NL and B2GP-PLYP-NL given there differ slightly from those obtained earlier ${ }^{16}$ from calculations uncorrected for basis set superposition error; even with the def2QZVP basis set, the basis set superposition error in a double hybrid is significant enough that this makes a difference for $b$ of as much as one unit. ${ }^{91}$

Symmetry-adapted perturbation theory calculations were carried out using the implementation (ref. 39 and references therein) in a prerelease version of PSI $4 .{ }^{92}$

For the purposes of basis set extrapolation, we employed a two-point expression of the form $E(L)=E_{\infty}+[E(L)-$ $E(L-1)] /\left[\left(\frac{L}{L-1}\right)^{\alpha}-1\right]$, in which $\alpha$ is taken from Table 2. We wish to point out that all of the various expressions for twopoint extrapolation are mathematically equivalent (see, e.g., ref. 93), and have merely converted them to a single form for convenience. Extrapolations of SCF and correlation energies were always performed separately; in the F12 calculations, the SCF component was taken from the largest basis set calculation.

The cc-pV\{Q,5\}Z-F12 extrapolation exponents in the present work were obtained by following, to the letter, the optimization procedure for the cc-pV\{T,Q\}Z-F12 exponents detailed in ref. 48 .

\section{Calibration of the reference method}

\section{Choice of the MP2-F12 reference level}

For the smaller, earlier, S22 dataset of noncovalent interactions, a revised set of benchmark data was reported by Marshall, Burns, and Sherrill (MBS). ${ }^{12}$ Aside from total $\operatorname{CCSD}(\mathrm{T})$ limit interaction energies given in the paper itself, HLCs (high-level corrections) are given in Table S1 of that paper's ESI, and MP2 limits were extracted as the difference. These correspond to counterpoisecorrected $\mathrm{AV}\{\mathrm{Q}, 5\} \mathrm{Z}$ basis set extrapolation.

Thus, we are able to consider performance for the MP2 basis set limit and for the HLC in isolation.

Our counterpoise-corrected DF-MP2-F12/cc-pVQZ-F12 interaction energies differ from the MBS estimated MP2 limits by just $0.01 \mathrm{kcal} \mathrm{mol}^{-1}$. This increases to $0.02 \mathrm{kcal} \mathrm{mol}^{-1}$ upon cc-pV\{T,Q\}Z-F12 extrapolation: for the corresponding raw and half-counterpoise values, RMSDs are 0.053 and $0.036 \mathrm{kcal} \mathrm{mol}^{-1}$, respectively.

Calculating DF-MP2-F12/cc-pVTZ-F12 and DF-MP2-F12/ cc-pVQZ-F12 energies for the entire S66x8 set proved technically quite feasible, both with and without counterpoise correction. We may conclude that the MP2 component is not the accuracylimiting factor.

In previous studies ${ }^{25,27}$ where comparison with the even larger cc-pV5Z-F12 basis set $^{27}$ was possible, it was concluded that half-counterpoise came closest to the basis set limit.

With considerable effort, we were able to obtain DF-MP2F12/cc-pV5Z-F12 interaction energies for the $1.0 r_{\mathrm{e}}$ slice of S66x8 (which has slightly different geometries than the S66 set particularly for $\pi$ stacks). The RMS counterpoise correction at that level is just $0.010 \mathrm{kcal} \mathrm{mol}^{-1}$. Between raw and counterpoise cc-pV\{Q,5\}Z-F12 extrapolated values - which should ideally be identical - the RMS difference drops even further to $0.004 \mathrm{kcal} \mathrm{mol}^{-1}$. (As noted in the Methods section, the extrapolation exponent in Table 2, 5.0723, was obtained in the present work, and the MP2-F12 correlation component was extrapolated in isolation and combined with the largest basis set $\mathrm{HF}+\mathrm{CABS}$ component. For the smaller cc-pV\{T,Q\}Z-F12 sequence, the extrapolation exponent 4.3548 was taken from ref. $48, c f$. Table 2.)

Error statistics for smaller basis sets can be found in Table 3 . The counterpoise-corrected $\{\mathrm{T}, \mathrm{Q}\}-\mathrm{F} 12$ values deviate from the corresponding $\{\mathrm{Q}, 5\}-\mathrm{F} 12$ limits by just $0.004 \mathrm{kcal} \mathrm{mol}^{-1} \mathrm{RMS}$; for half-counterpoise, this increases to $0.013 \mathrm{kcal} \mathrm{mol}^{-1}$ relative to counterpoise $\{\mathrm{Q}, 5\}-\mathrm{F} 12$, or $0.014 \mathrm{kcal} \mathrm{mol}^{-1}$ relative to halfcounterpoise $\{\mathrm{Q}, 5\}$-F12 limits. If we were to dispense with extrapolation entirely, half-counterpoise cc-pVQZ-F12 appears 
Table 3 RMSD ( $\mathrm{kcal} \mathrm{mol}^{-1}$ ) for the MP2-F12 limits of the $1.0 r_{\mathrm{e}}$ slice of S66x8, relative to cc-pV\{Q,5\}Z-F12 results

cc-pVTZ-F12 cc-pVQZ-F12 cc-pV5Z-F12 cc-pV\{T,Q\}Z-F12 cc-pV\{Q,5\}Z-F12 MP2/AV\{T,Q\}Z

relative to $\mathrm{V}\{\mathrm{Q}, 5\} \mathrm{Z}-\mathrm{F} 12$ raw

\begin{tabular}{|c|c|c|c|c|c|c|}
\hline Raw & 0.073 & 0.035 & 0.009 & 0.029 & REF & \\
\hline $\mathrm{CP}$ & 0.050 & 0.013 & 0.004 & 0.006 & 0.004 & 0.015 \\
\hline Half & 0.017 & 0.013 & 0.004 & 0.016 & 0.002 & \\
\hline \multicolumn{7}{|c|}{ relative to $\mathrm{V}\{\mathrm{Q}, 5\} \mathrm{Z}-\mathrm{F} 12 \mathrm{CP}$} \\
\hline Raw & 0.070 & 0.032 & 0.006 & 0.026 & 0.004 & \\
\hline $\mathrm{CP}$ & 0.053 & 0.015 & 0.005 & 0.004 & REF & 0.017 \\
\hline Half & 0.014 & 0.010 & 0.001 & 0.013 & 0.002 & \\
\hline \multicolumn{7}{|c|}{ relative to $\mathrm{V}\{\mathrm{Q}, 5\} \mathrm{Z}-\mathrm{F} 12$ half } \\
\hline Raw & 0.071 & 0.033 & 0.007 & 0.027 & 0.002 & \\
\hline $\mathrm{CP}$ & 0.051 & 0.014 & 0.004 & 0.004 & 0.002 & 0.016 \\
\hline Half & 0.015 & 0.011 & 0.003 & 0.014 & REF & \\
\hline
\end{tabular}

to have a slight edge over full counterpoise, and a definite one over the raw values: in fact, half-counterpoise cc-pVTZ-F12 is found to be preferred over raw cc-pVQZ-F12.

We finally chose full-counterpoise MP2-F12/cc-pV\{T,Q\}Z-F12 extrapolation as the MP2 component for our benchmark data. Based on the statistics given in Table 3, we conservatively estimate the accuracy of our MP2 limits to be better than $0.01 \mathrm{kcal} \mathrm{mol}^{-1}$.

For comparison, the conventional, counterpoise-corrected MP2/aug-cc-pV $\{\mathrm{T}, \mathrm{Q}\} \mathrm{Z}$ values used by the Hobza group as the MP2 component of the S66x8 benchmark data in the Benchmark Energy and Geometry Database (www.begdb.com) ${ }^{14}$ were recalculated in the present work. They differ from our best reference values by $0.017 \mathrm{kcal} \mathrm{mol}^{-1} \mathrm{RMS}$.

\section{Choice of the high-level correction, i.e., $\operatorname{CCSD}(\mathrm{T})-\mathrm{MP} 2$ contribution}

The S22 dataset as a model. We shall first revisit the S22 set. For some of the larger systems, CCSD(T)-F12b/cc-pVTZ-F12 turned out to be unfeasible in practice: this statement applies to both structures of the adenine $\cdots$ thymine base pair (WatsonCrick and stacked), both structures of indole * benzene (parallel and T-shaped), phenol dimer, and 2-pyridoxine $\cdots 2$-aminopyridine. We were unable to obtain a counterpoise correction for the stacked uracil dimer, but the uncorrected interaction energy ran to completion, taking more than a week on 32 CPUs.

The crucial factor affecting performance will be the choice of approximation to the (T) connected triple excitations (see Methods section). RMSDs for the HLCs of the S22 set are presented in Table 4.

For the subset, we find that HLC(Ts)/cc-pVTZ-F12 has an RMSD of less than $0.02 \mathrm{kcal} \mathrm{mol}^{-1}$ from the MBS reference data. With the said basis set, there is little to choose between $\operatorname{HLC}(\mathrm{Ts}), \operatorname{HLC}\left(\mathrm{Tb}_{\mathrm{sc}}\right)$, and $\operatorname{HLC}\left(\mathrm{T}^{*}{ }_{\mathrm{sc}}\right)$ - or the non-size-consistent variant $\mathrm{HLC}\left(\mathrm{T}^{*}\right)$, for that matter - as the difference between the RMSDs is less than the presumed uncertainty in the reference data.

Counterpoise correction on the HLC appears to be unhelpful, which is useful considering its computational cost for larger systems. (Counterpoise calculations typically require disabling symmetry.)
For the smaller cc-pVDZ-F12 basis sets, the raw HLCs are definitely preferred over counterpoise and half-counterpoise. Among the size-consistent options, $\left(\mathrm{Tb}_{\mathrm{sc}}\right)$ and $(\mathrm{Ts})$ seem to have an edge over $\left(\mathrm{T}^{*}{ }_{\mathrm{sc}}\right)$, with there again being little to choose between $\left(\mathrm{Tb}_{\mathrm{sc}}\right)$ and $(\mathrm{Ts})$. In our experience, ${ }^{27}$ with cc-pVTZ-F12 and larger basis sets, CCSD-F12b and the more rigorous $\operatorname{CCSD}\left(\mathrm{F} 12^{*}\right),{ }^{96}$ a.k.a. CCSD-F12c, method yield nearly identical results, but with the cc-pVDZ-F12 basis set, $\mathrm{CCSD}\left(\mathrm{F} 12^{*}\right)$ may offer an edge for some applications. ${ }^{25}$ For the S22 set, we cannot distinguish between the F12b and (F12*) approaches based on the RMSD for HLC alone. We do note, however, that the CCSD-MP2 parts of $\left(\mathrm{F} 12^{*}\right) / \mathrm{cc}-\mathrm{pVDZ}-\mathrm{F} 12$ are considerably closer $\left(\mathrm{RMSD}=0.019 \mathrm{kcal} \mathrm{mol}^{-1}\right)$ to the available F12b/cc-pVTZF12 values than the F12b/cc-pVDZ-F12 counterparts $(\mathrm{RMSD}=$ $0.044 \mathrm{kcal} \mathrm{mol}^{-1}$ ).

For the entire S22 set, conventionally computed HLCs with different basis sets are available from the ESI of ref. 12 and 97. The lowest RMSD from the MBS reference data, $0.04 \mathrm{kcal} \mathrm{mol}^{-1}$, is found for counterpoise-corrected $\operatorname{CCSD}(\mathrm{T}) /$ aug-cc-pV $\{\mathrm{D}, \mathrm{T}\} \mathrm{Z}$.

The S66x8 dataset and the 1.0r $r_{\mathrm{e}}$ slice thereof. Let us further consider, with the cc-pVDZ-F12 basis set, the differences between various triples corrections for the entire S66 88 set, using cc-pVDZ-F12. Size-consistency errors in $\operatorname{CCSD}\left(\mathrm{T}^{*}\right)$, i.e., with individual scaling for monomers and dimers, range from -0.12 to $+0.20 \mathrm{kcal} \mathrm{mol}^{-1}$, clearly unacceptable for our purposes. The difference between $\mathrm{Tb}_{\mathrm{sc}}$ and $\mathrm{Ts}$, on the other hand, is just $0.015 \mathrm{kcal} \mathrm{mol}^{-1} \mathrm{RMSD}$, with a maximum of $0.086 \mathrm{kcal} \mathrm{mol}^{-1}$ for system 26, stacked uracil dimer at $0.90 r_{\mathrm{e}}$. (At the equilibrium geometry, this drops to $0.060 \mathrm{kcal} \mathrm{mol}^{-1}$.) For the same system, the largest difference $\left(-0.054 \mathrm{kcal} \mathrm{mol}^{-1}\right)$ is also seen between the $\left(\mathrm{Tc}_{\mathrm{sc}}\right)$ obtained from $\mathrm{CCSD}\left(\mathrm{F} 12^{*}\right)$ and the $\left(\mathrm{Tb}_{\mathrm{sc}}\right)$ from CCSDF12b. On average, $\mathrm{CCSD}\left(\mathrm{F} 12^{*}\right)$ amplitudes result in triples corrections that are systematically smaller $\left(-0.01 \mathrm{kcal} \mathrm{mol}^{-1}\right)$ than those from CCSD-F12b.

Let us consider the CCSD-MP2 differences with the cc-pVDZ-F12 basis set in isolation. Here we do not have to contend with the different (T) options: our two available choices are $\operatorname{CCSD}\left(\mathrm{F} 12^{*}\right)$ MP2-F12 and CCSD-F12b-MP2-F12. The most significant differences between them are seen for systems with multiple hydrogen 
Table 4 RMSD ( $\mathrm{kcal} \mathrm{mol}^{-1}$ ) for the high-level corrections HLC [(CCSD(T)-F12-MP2-F12)/cc-pVnZ-F12] of a subset of S22, relative to the MBS reference data

\begin{tabular}{rllllll} 
& HLC(T*) & HLC(Ts) & HLC(T) & HLC( $\left(_{\text {sc }}^{*}\right)$ & HLC(Tb) & HLC(Tb sc $)$ \\
\hline F12b/cc-pVDZ-F12 Raw & 0.049 & 0.047 & 0.128 & 0.044 & 0.055 & 0.041 \\
CP & 0.126 & 0.171 & 0.244 & 0.143 & 0.138 & 0.158 \\
Half & 0.080 & 0.103 & 0.184 & 0.075 & 0.090 & 0.090 \\
\hline F12*)/cc-pVDZ-F12 Raw & 0.053 & 0.041 & 0.096 & 0.063 & 0.052 & 0.052 \\
\hline F12b/cc-pVTZ-F12 Raw & 0.024 & 0.017 & 0.043 & 0.025 & 0.018 & 0.018 \\
CP & 0.051 & 0.071 & 0.105 & 0.054 & 0.063 & 0.063 \\
Half & 0.031 & 0.038 & 0.073 & 0.022 & 0.030 & 0.030
\end{tabular}

bonds, such as 17 (uracil dimer Watson-Crick), 20 (acetic acid dimer), 21 (acetamide dimer), 22 (acetic acid-uracil), and 23 (acetamide-uracil), for each of which the difference exceeds $0.100 \mathrm{kcal} \mathrm{mol}^{-1}$ at equilibrium or compressed geometries. As expected, these differences are greatly reduced for stretched geometries and effectively dwindle to nothing at $2.0 r_{\mathrm{e}}$.

(F12*)-F12b differences are smaller, but still some what significant, for $\pi$ stacks and mixed-influence complexes, but effectively negligible for London complexes.

Which is more correct? At great computational expense and following multiple failures due to Linux kernel tuning issues, we were able to perform CCSD(T)-F12b/cc-pVTZ-F12 calculations for a subset of 58 out of 66 systems at the $1.0 r_{\mathrm{e}}$ geometries. The subset consists of all systems except the London complexes 35 and 37-43. The CCSD-MP2 differences in the dissociation energies, obtained as a by-product, should be quite close to the basis set limit. The [CCSD(F12*)-MP2-F12]/cc-pVDZ-F12 agrees with those to $0.013 \mathrm{kcal} \mathrm{mol}^{-1} \mathrm{RMS}$ (Table 5), compared to $0.040 \mathrm{kcal} \mathrm{mol}^{-1}$ for [CCSD-F12b-MP2-F12]/cc-pVDZ-F12. We have hence decided to err on the side of rigor, and to favor $\operatorname{CCSD}\left(\mathrm{F} 12^{*}\right)$ over CCSD-F12b, as we have at present no realistic prospect of carrying out CCSD-F12b/cc-pVTZ-F12 calculations for the entire S66x8 dataset.
Performance of different (T) scaling procedures for the HLCs of the 58-system subset has been compared in Table 5 . First, we note that they all yield very similar values for the cc-pVTZ-F12 basis set: $\mathrm{HLC}\left(\mathrm{Tb}_{\mathrm{sc}}\right)$ differs from $\mathrm{HLC}(\mathrm{Ts})$ by less than $0.01 \mathrm{kcal} \mathrm{mol}^{-1} \mathrm{RMS}$, and by just $0.013 \mathrm{kcal} \mathrm{mol}^{-1}$ from $\operatorname{HLC}\left(\mathrm{T}^{*}{ }_{\mathrm{sc}}\right)$. As generally $\operatorname{HLC}(\mathrm{Ts})<\operatorname{HLC}\left(\mathrm{Tb}_{\mathrm{sc}}\right)<\operatorname{HLC}\left(\mathrm{T}^{*}{ }_{\mathrm{sc}}\right)$, we have somewhat arbitrarily selected the middle values $\operatorname{HLC}\left(\mathrm{Tb}_{\mathrm{sc}}\right) / \mathrm{cc}-\mathrm{pVTZ}-\mathrm{F} 12$ for comparison, but another choice would not have led to qualitatively different conclusions.

Second, $\quad \operatorname{HLC}\left(\mathrm{Tb}_{\mathrm{sc}}\right) / \mathrm{cc}-\mathrm{pVDZ}-\mathrm{F} 12, \quad \mathrm{HLC}\left(\mathrm{Tc}_{\mathrm{sc}}\right) / \mathrm{cc}-\mathrm{pVDZ}-\mathrm{F} 12$, and (Ts) from $\left(\mathrm{F} 12^{*}\right) / \mathrm{cc}-\mathrm{pVDZ}-\mathrm{F} 12$ or F12b/cc-pVDZ-F12 all agree with those values to $0.025-0.042 \mathrm{kcal} \mathrm{mol}^{-1} \mathrm{RMS}$, with a possible slight advantage for $\left(\mathrm{F} 12^{*}\right) / \mathrm{cc}-\mathrm{pVDZ}-\mathrm{F} 12$ over $\mathrm{F} 12 \mathrm{~b} /$ cc-pVDZ-F12. The RMSDs of HLC(Ts)(F12*)/cc-pVDZ-F12 and $\mathrm{HLC}\left(\mathrm{Tc}_{\mathrm{sc}}\right)\left(\mathrm{F} 12^{*}\right) / \mathrm{cc}-\mathrm{pVDZ}-\mathrm{F} 12$ however are essentially identical, hence no meaningful choice between them can be made from the numbers alone. On the other hand, while (Ts) contains a single empirical parameter (the 'one size fits all' scaling factor), $\left(\mathrm{Tb}_{\mathrm{sc}}\right)$ and $\left(\mathrm{Tc}_{\mathrm{sc}}\right)$ contain none at all and instead elicit everything from $a b$ initio calculations for the actual system at hand. For want of a realistic option to use larger basis sets, we therefore decided on $\operatorname{HLC}\left(\mathrm{Tc}_{\mathrm{sc}}\right) / \mathrm{cc}-\mathrm{pVDZ}-\mathrm{F} 12$, i.e., with the triples scaled by the $E_{\text {corr }}\left[\mathrm{CCSD}\left(\mathrm{F} 12^{*}\right)\right] / E_{\text {corr }}[\mathrm{CCSD}]$ ratio, as our final choice for the

Table 5 RMS differences ( $\left(\mathrm{ccal} \mathrm{mol}^{-1}\right.$ ) between various high-level corrections with the cc-pVDZ-F12 and cc-pVTZ-F12 basis sets for a 58 -system subset of the $1.0 r_{\mathrm{e}}$ slice

\begin{tabular}{|c|c|c|c|c|c|}
\hline & $\operatorname{HLC}\left(\mathrm{T}^{*}\right)$ & HLC(T) & $\mathrm{HLC}\left(\mathrm{Tb}_{\mathrm{sc}}\right)$ & HLC(Ts) & $\mathrm{HLC}\left(\mathrm{T}_{\mathrm{sc}}^{*}\right)$ \\
\hline \multicolumn{6}{|c|}{ relative to $\mathrm{HLC}\left(\mathrm{Tb}_{\mathrm{sc}}\right) / \mathrm{cc}-\mathrm{pVTZ}-\mathrm{F} 12$} \\
\hline F12b/cc-pVTZ-F12 & 0.021 & 0.052 & REF & 0.009 & 0.013 \\
\hline$\left(\mathrm{F} 12^{*}\right) / \mathrm{VDZ}-\mathrm{F} 12$ & 0.045 & 0.116 & 0.031 & 0.025 & 0.042 \\
\hline F12b/cc-pVDZ-F12 & 0.042 & 0.136 & 0.027 & 0.036 & 0.030 \\
\hline \multicolumn{6}{|c|}{ relative to HLC(Ts)/cc-pVTZ-F12 } \\
\hline F12b/cc-pVTZ-F12 & 0.021 & 0.043 & 0.009 & REF & 0.022 \\
\hline$\left(\mathrm{F} 12^{*}\right) / \mathrm{VDZ}-\mathrm{F} 12$ & 0.046 & 0.108 & 0.036 & 0.025 & 0.050 \\
\hline F12b/cc-pVDZ-F12 & 0.039 & 0.127 & 0.026 & 0.030 & 0.036 \\
\hline \multicolumn{6}{|c|}{ relative to HLC(T $\left.{ }_{\text {sc }}^{*}\right) / c c-p V T Z-F 12$} \\
\hline F12b/cc-pVTZ-F12 & 0.027 & 0.065 & 0.013 & 0.022 & REF \\
\hline$\left(\mathrm{F} 12^{*}\right) / \mathrm{VDZ}-\mathrm{F} 12$ & 0.048 & 0.129 & 0.028 & 0.032 & 0.034 \\
\hline F12b/cc-pVDZ-F12 & 0.050 & 0.149 & 0.034 & 0.046 & 0.026 \\
\hline
\end{tabular}

CCSD-MP2 relative to cc-pVTZ-F12

F12b/cc-pVTZ-F12 REF

$\left(\mathrm{F} 12^{*}\right) / \mathrm{VDZ}-\mathrm{F} 12$

F12b/cc-pVDZ-F12 $\quad 0.040$ 
reference data. For the 58-system subset, the $\operatorname{HLC}\left(\mathrm{Tc}_{\mathrm{sc}}\right) /$ cc-pVDZ-F12 combination agrees to $0.031 \mathrm{kcal} \mathrm{mol}^{-1} \mathrm{RMS}$ with the cc-pVTZ-F12 data, compared to $0.09 \mathrm{kcal} \mathrm{mol}^{-1}$ for the counterpoised conventional [CCSD(T)-MP2]/aug-cc-pVDZ HLCs used in the original $\mathrm{S} 66 \times 8$ paper, which we reconstructed by recalculating their counterpoised MP2/aug-cc-pV $\{\mathrm{T}, \mathrm{Q}\} \mathrm{Z}$ components and subtracting from the total $\mathrm{S} 66 \mathrm{x} 8$ values.

\section{Counterpoise corrections for lower-level methods}

We then are faced with not only the choice of basis set for the lower-level methods, but also the choice of counterpoise correction. It is fairly well-known (e.g., ref. 98) that, for noncovalent interactions, uncorrected dissociation energies approach the basis set limit from above, and counterpoise-corrected ones from below: the halfhalf counterpoise method, i.e., the average of raw and counterpoise interaction energies, then immediately suggests itself. In ref. 99 it was shown that half-counterpoise generally comes closest to the basis set limit for orbital-based wavefunction $a b$ initio calculations. In ref. 26 we showed that this is also generally the case for explicitly correlated methods, except perhaps for large basis sets like cc-pVQZ-F12 and especially cc-pV5Z-F12, where full counterpoise may be more appropriate but the counterpoise corrections in any case become insignificant.

At the HF level, things are rather different. For def2-TZVPP, half-counterpoise appears to have an edge over full counterpoise, but for all larger basis sets full counterpoise "carries the day", with an RMSD as small as $0.005 \mathrm{kcal} \mathrm{mol}^{-1}$ for the haVQZ basis set, and $0.013 \mathrm{kcal} \mathrm{mol}^{-1}$ for def2-QZVP (Table 6). It hardly matters whether one uses the CABS-corrected HF/cc-pVQZ-F12 or the orbital $\mathrm{HF} / \mathrm{haV} 5 \mathrm{Z}$ as references: the counterpoise-corrected values for both differ by no more than $0.002 \mathrm{kcal} \mathrm{mol}^{-1} \mathrm{RMS}$.

As can be seen in Table 6, HF/def2-QZVP with full counterpoise is quite close to the basis set limit, HF/haVQZ even closer.

What about DFT functionals below rung five? We considered the example of PBE0. Somewhat arbitrarily, we chose haV5Z with full counterpoise correction as the reference: the difference with half-counterpoise in the same basis set amounts to just $0.005 \mathrm{kcal} \mathrm{mol}^{-1}$ RMSD (Table 7). For essentially all basis sets considered, full counterpoise is clearly the best of the three options, except that for def2-QZVPD' the gap with half-counterpoise is quite narrow. At any rate, the RMSD of $0.02 \mathrm{kcal} \mathrm{mol}^{-1}$ for
def2-QZVP is considered small enough that we can use it for benchmark purposes.

Summing up: for Hartree-Fock and DFT functionals, we will use full counterpoise and a basis of def2-QZVP or better quality.

For MP2, it appears even haVQZ is not adequate to reach the basis set limit, but haV\{T,Q\}Z extrapolation with full counterpoise does succeed. Half-counterpoise haV5Z comes reasonably close without extrapolation, as does $\operatorname{haV}\{\mathrm{Q}, 5\} \mathrm{Z}$ without counterpoise. In contrast, with the explicitly correlated approach, even MP2-F12/cc-pVTZ-F12 is already within $0.01 \mathrm{kcal} \mathrm{mol}^{-1} \mathrm{RMS}$ if half-counterpoise is applied (Table 8). At any rate, as reported by Burns et al. ${ }^{99}$ for conventional correlated calculations and our group $^{26}$ for explicitly correlated ones, half-counterpoise is unambiguously preferred.

This finally leaves the double hybrids, where one might expect behavior to be intermediate between MP2 and PBE0. For basis sets def2-QZVP and larger, we can expect the Kohn-Sham part to be converged, which leaves the MP2-like component as the dominant factor in basis set convergence for double hybrids.

Based on the results given in Table 9, we have selected halfcounterpoise with the haVQZ basis set as our basis set of choice, with def2-QZVP with half-counterpoise as a fallback. With smaller Weigend-Ahlrichs ${ }^{41}$ basis sets, half-counterpoise is again unequivocally preferred, justifying the design decisions taken in ref. 79, 80 and 84.

\section{Results and discussion}

\section{Wavefunction $a b$ initio results}

Since so much work on weak interactions has historically focused on hydrogen bonds (and, to a lesser extent, London dispersion), it is received wisdom in much of the quantum chemical community that MP2 is a "high-level ab initio" treatment for noncovalent interactions. In fact, for the S66x8 dataset with the cc-pVQZ-F12 basis set (half counterpoise), the RMSD is a somewhat disappointing $0.69 \mathrm{kcal} \mathrm{mol}^{-1}$ (Table 10). Some things become clearer if we break the results down by categories: hydrogen bonds (systems 1-23), $\pi$ stacks (systems 24-33), London dispersion complexes (systems 34-46), and mixed-influence (systems 47-66). We then see that MP2 has an excellent RMSD = $0.18 \mathrm{kcal} \mathrm{mol}^{-1}$ for hydrogen bonds, a tolerable $0.40 \mathrm{kcal} \mathrm{mol}^{-1}$ for

Table 6 RMSD ( $\mathrm{kcal} \mathrm{mol}^{-1}$ ) for the Hartree-Fock components of the S66x8 interaction energies as calculated with various basis sets

\begin{tabular}{|c|c|c|c|c|c|c|}
\hline & \multicolumn{3}{|c|}{ w.r.t. $\mathrm{HF}+\mathrm{CABS} / \mathrm{cc}-\mathrm{pVQZ}-\mathrm{F} 12 \mathrm{CP}$} & \multicolumn{3}{|c|}{ w.r.t. HF/haV5Z CP } \\
\hline & $\mathrm{CP}$ & Raw & Half & CP & Raw & Half \\
\hline haVQZ & 0.005 & 0.026 & 0.012 & 0.005 & 0.026 & 0.012 \\
\hline haVTZ & 0.028 & 0.110 & 0.051 & 0.028 & 0.110 & 0.051 \\
\hline def2-QZVP & 0.013 & 0.063 & 0.031 & 0.014 & 0.064 & 0.031 \\
\hline haV5Z & 0.002 & 0.008 & 0.004 & REF & 0.008 & 0.004 \\
\hline def2-TZVPP & 0.046 & 0.229 & 0.012 & 0.046 & 0.229 & 0.011 \\
\hline def2-TZVP & 0.110 & 0.298 & 0.132 & 0.110 & 0.298 & 0.132 \\
\hline VQZ-F12+CABS & REF & 0.008 & 0.004 & 0.002 & 0.008 & 0.004 \\
\hline
\end{tabular}


Table 7 RMSD ( $\mathrm{kcal} \mathrm{mol}^{-1}$ ) for the interaction energies of $\mathrm{S} 66 \times 8$ set calculated with $\mathrm{PBEO}$ using various basis sets relative to counterpoise corrected PBEO/haV5Z

\begin{tabular}{llll}
\hline Counterpoise & CP & Raw & Half \\
\hline haV5Z & REF & 0.009 & 0.005 \\
haVQZ & 0.005 & 0.046 & 0.151 \\
haVTZ & 0.027 & 0.093 & 0.046 \\
def2-QZVP & 0.020 & 0.105 & 0.054 \\
def2-TZVPP & 0.069 & 0.310 & 0.157 \\
def2-TZVP & 0.143 & 0.392 & 0.234 \\
def2-QZVPD' & 0.010 & 0.032 & 0.015 \\
def2-TZVPPD' & 0.028 & 0.086 & 0.038 \\
def2-TZVPD' & 0.074 & 0.100 & 0.122 \\
aug'-pc2 & 0.025 & 0.109 & 0.051 \\
aug'-pc3 (partial results) & 0.003 & 0.010 & 0.005
\end{tabular}

Table 8 RMSD ( $\mathrm{kcal} \mathrm{mol}^{-1}$ ) for the interaction energies of the $\mathrm{S} 66 \times 8$ set relative to half-counterpoise corrected MP2-F12/cc-pV\{T,Q\}Z-F12

\begin{tabular}{llll}
\hline Counterpoise & Raw & CP & Half \\
\hline MP2-F12/cc-pVTZ-F12 & 0.058 & 0.045 & 0.012 \\
MP2-F12/cc-pVQZ-F12 & 0.024 & 0.012 & 0.007 \\
MP2-F12/cc-pV\{T,Q\}Z-F12 & 0.018 & REF & 0.009 \\
MP2/haVTZ & 0.461 & 0.346 & 0.162 \\
MP2/haVQZ & 0.170 & 0.150 & 0.058 \\
MP2/haV5Z & 0.073 & 0.079 & 0.026 \\
MP2/haV\{T,Q\}Z & 0.047 & 0.018 & 0.023 \\
MP2/haV\{Q,5\}Z & 0.028 & 0.011 & 0.017 \\
MP2/def2-QZVP & 0.158 & 0.289 & 0.084
\end{tabular}

London complexes, and an appalling $1.54 \mathrm{kcal} \mathrm{mol}^{-1}$ for $\pi$ stacks. It has been known for some time (e.g., ref. 100 and 101) that $\pi-\pi$ interactions at the MP2 level are severely overestimated due to the dispersion component of the 2nd-order energy effectively corresponding to uncoupled Hartree-Fock dispersion (ref. 102; see also ref. 103).
SCS-MP2 mostly remedies the issue for $\pi$ stacks, at the expense of degraded performance for London complexes and especially hydrogen bonds. SCS(MI)MP2, optimized for weak interactions, yields fairly poor results for London complexes, but very good to excellent results for the rest of S66x8, at the expense of general thermochemistry. The quasi-first principles S2-MP2 ${ }^{56}$ trades off some of the great performance for hydrogen bonds for better results in the other categories, still yielding unacceptable pi complexes. Similar to a wrinkle in a carpet larger than the room, the error of parametrized MP2 can be moved from one category to another, but never fully removed. Ad hoc fitting of SCS-MP2 yielded $c_{2 \mathrm{ab}}=0.339$, $c_{2 \mathrm{ss}}=1.429, \mathrm{RMSD}=0.285 \mathrm{kcal} \mathrm{mol}^{-1}$, somewhat better than the similar SCS(MI)MP2.

Moving on to higher-cost methods, third-order corrections $E_{3}$ were evaluated in $\mathrm{MP} 3\left(\mathrm{~F} 12^{*}\right) / \mathrm{cc}-\mathrm{pVDZ}-\mathrm{F} 12$ calculations using Turbomole and added in to half-counterpoise MP2-F12/ cc-pVQZ-F12 and spin-component-scaled variants thereof. SCSMP3 in fact is found to perform worse than SCS-MP2. MP2.5 on the other hand, ${ }^{104}$ - averaging between MP2 and MP3, which typically err on opposite sides of the true number - yields a rather pleasing RMSD $=0.21 \mathrm{kcal} \mathrm{mol}^{-1}$, with especially outstanding performance for $\mathrm{H}$-bonds and mixed-influence complexes, while $\pi$ stacks are still acceptable at $0.44 \mathrm{kcal} \mathrm{mol}^{-1}$. Ad hoc refitting of $c_{3}$ yields a very modest further improvement in RMSD, while additionally refitting $c_{2 s s}$ and $c_{2 \mathrm{ab}}$ is found to be statistically insignificant.

As an aside, Hesselmann proposed ${ }^{105}$ the MP2C method, in which the dispersion part was removed from MP2 and replaced by its TDDFT counterpart. Basis set limit extrapolated MP2C values for the $\mathrm{S} 66$ set (i.e., just the equilibrium geometry slice of S66x8) have been reported in ref. 106: the RMSD from their reference data was given as $0.13 \mathrm{kcal} \mathrm{mol}^{-1}$.

Turning now to $\operatorname{CCSD}\left(\mathrm{F} 12^{*}\right)$, we find that it actually performs worse than MP2-F12. SCS-CCSD(F12*) greatly improves things, and SCS(MI)CCSD(F12*) especially so (Table 11). Ad hoc minimization of RMSD with respect to $c_{\mathrm{ss}}$ and $c_{\mathrm{ab}}$ yields coefficients fairly close to $\operatorname{SCS}(\mathrm{MI}) \operatorname{CCSD}\left(\mathrm{F} 12^{*}\right)$, yet simply scaling the $\operatorname{CCSD}\left(\mathrm{F} 12^{*}\right)$ correlation energy by a factor of 1.23 seems to

Table 9 RMSD ( $\mathrm{kcal} \mathrm{mol}^{-1}$ ) for the interaction energies of S66 8 set calculated with the DSD-PBEP86-D3BJ method using various basis sets

\begin{tabular}{|c|c|c|c|c|c|c|c|c|c|}
\hline & \multicolumn{3}{|c|}{ Relative to haV5Z(CP) } & \multicolumn{3}{|c|}{ Relative to haV5Z(half) } & \multicolumn{3}{|c|}{ Relative to haV5Z(raw) } \\
\hline & $\mathrm{CP}$ & Raw & Half & $\mathrm{CP}$ & Raw & Half & CP & Raw & Half \\
\hline $\mathrm{haV}\{\mathrm{Q}, 5\} \mathrm{Z}$ & 0.036 & 0.030 & 0.030 & 0.017 & 0.036 & 0.025 & 0.046 & 0.068 & 0.057 \\
\hline haV5Z & REF & 0.076 & 0.038 & 0.038 & 0.038 & REF & 0.076 & REF & 0.038 \\
\hline haVQZ & 0.034 & 0.138 & 0.056 & 0.070 & 0.101 & 0.024 & 0.108 & 0.065 & 0.030 \\
\hline haVTZ & 0.130 & 0.322 & 0.122 & 0.165 & 0.286 & 0.095 & 0.201 & 0.251 & 0.078 \\
\hline def2-QZVPD' & 0.075 & 0.172 & 0.059 & 0.112 & 0.136 & 0.037 & 0.149 & 0.101 & 0.046 \\
\hline def2-QZVP & 0.102 & 0.163 & 0.041 & 0.139 & 0.128 & 0.032 & 0.176 & 0.095 & 0.057 \\
\hline def2-TZVPPD' & 0.199 & 0.353 & 0.123 & 0.235 & 0.319 & 0.106 & 0.272 & 0.285 & 0.102 \\
\hline def2-TZVPP & 0.298 & 0.348 & 0.102 & 0.335 & 0.315 & 0.106 & 0.372 & 0.284 & 0.123 \\
\hline def2-TZVPD & 0.281 & 0.380 & 0.157 & 0.317 & 0.349 & 0.155 & 0.352 & 0.319 & 0.161 \\
\hline def2-TZVP & 0.401 & 0.381 & 0.203 & 0.436 & 0.355 & 0.216 & 0.472 & 0.332 & 0.234 \\
\hline
\end{tabular}


Table 10 Error statistics ( $\mathrm{kcal} \mathrm{mol}^{-1}$ ) for the S66x8 interaction energies calculated at the half-counterpoise MP2-F12/cc-pVQZ-F12 level and spincomponent scaled variants thereof, as well as with raw [MP3-F12-MP2-F12]/cc-pVDZ-F12 terms added

\begin{tabular}{|c|c|c|c|c|c|c|c|}
\hline & MP2-F12 & SCS-MP2-F12 & SCS(MI)MP2-F12 & 2 S2-MP2-F12 & SCS-MP3-F12 & MP2.5 & $\begin{array}{l}\text { Ad hoc } \\
\text { MP2.Xopt }\end{array}$ \\
\hline MSD all & 0.293 & -0.541 & -0.178 & 0.102 & -0.694 & -0.012 & -0.046 \\
\hline H-bonds & -0.051 & -0.732 & -0.166 & -0.246 & -0.753 & -0.093 & -0.096 \\
\hline$\pi$ stacks & 1.119 & -0.240 & 0.049 & 0.852 & -0.680 & 0.238 & 0.141 \\
\hline London & 0.228 & -0.698 & -0.475 & 0.042 & -0.868 & -0.112 & -0.150 \\
\hline Mixed & 0.319 & -0.371 & -0.113 & 0.168 & -0.520 & 0.021 & -0.012 \\
\hline H-bonds & 0.184 & 0.967 & 0.222 & 0.393 & 0.972 & 0.122 & 0.125 \\
\hline$\pi$ stacks & 1.536 & 0.527 & 0.233 & 1.226 & 0.887 & 0.442 & 0.369 \\
\hline London & 0.397 & 0.881 & 0.615 & 0.256 & 1.090 & 0.188 & 0.223 \\
\hline Mixed & 0.490 & 0.477 & 0.237 & 0.335 & 0.654 & 0.108 & 0.095 \\
\hline$c_{S S}$ & 1.000 & $1 / 3$ & 1.290 & 0.750 & $1 / 3$ & 1.000 & 1.000 \\
\hline
\end{tabular}

Table $11 \mathrm{~S} 66 \times 8$ error statistics $\left(\mathrm{kcal} \mathrm{mol}^{-1}\right)$ for $\operatorname{CCSD}\left(\mathrm{F} 12^{*}\right)$ and spin-component-scaled variants thereof, as well as for CCSD(T)(F12*) and $\operatorname{CCSD}\left(T^{*}\right)\left(\mathrm{F} 12^{*}\right)$

\begin{tabular}{|c|c|c|c|c|c|c|c|c|}
\hline & $\begin{array}{l}\text { SCS(Adhoc) } \\
\text { CCSD(F12*) }\end{array}$ & $\begin{array}{l}\text { SCS- } \\
\operatorname{CCSD}\left(\mathrm{F} 12^{*}\right)\end{array}$ & $\begin{array}{l}\operatorname{SCS}(\mathrm{MI}) \\
\operatorname{CCSD}\left(\mathrm{F} 12^{*}\right)\end{array}$ & $\begin{array}{l}\text { Scaled } \\
\operatorname{CCSD}\left(\mathrm{F} 12^{*}\right)\end{array}$ & $\begin{array}{l}\operatorname{CCSD}(\mathrm{T}) \\
\left(\mathrm{F} 12^{*}\right)\end{array}$ & $\begin{array}{l}\operatorname{CCSD}\left(\mathrm{T}^{*}\right) \\
\left(\mathrm{F} 12^{*}\right)\end{array}$ & $\begin{array}{l}\mathrm{CCSD} \\
\left(\mathrm{F} 12^{*}\right)\end{array}$ & $\begin{array}{l}\operatorname{CCSD}\left(\mathrm{F} 12^{*}\right) \\
-\mathrm{D} 2^{\mathrm{a}}\end{array}$ \\
\hline MSD all & 0.003 & -0.134 & -0.052 & -0.016 & -0.084 & -0.007 & -0.586 & -0.014 \\
\hline H-bonds & 0.020 & -0.177 & -0.026 & -0.054 & -0.067 & 0.011 & -0.440 & -0.085 \\
\hline$\pi$ stacks & -0.088 & -0.207 & -0.171 & -0.058 & -0.144 & -0.044 & -1.003 & -0.119 \\
\hline London & 0.032 & -0.068 & -0.035 & 0.052 & -0.094 & -0.038 & -0.688 & 0.130 \\
\hline Mixed & 0.012 & -0.090 & -0.034 & 0.004 & -0.068 & 0.013 & -0.479 & 0.027 \\
\hline RMSD all & 0.09 & 0.21 & 0.11 & 0.12 & 0.11 & 0.05 & 0.79 & 0.16 \\
\hline H-bonds & 0.06 & 0.28 & 0.07 & 0.14 & 0.08 & 0.03 & 0.56 & 0.18 \\
\hline$\pi$ stacks & 0.13 & 0.28 & 0.22 & 0.12 & 0.19 & 0.06 & 1.29 & 0.16 \\
\hline London & 0.13 & 0.14 & 0.12 & 0.15 & 0.12 & 0.05 & 0.86 & 0.20 \\
\hline Mixed & 0.04 & 0.10 & 0.05 & 0.05 & 0.09 & 0.05 & 0.61 & 0.07 \\
\hline $\mathrm{c}_{\mathrm{SS}}$ & 1.312 & 1.130 & 1.280 & 1.230 & 1 & 1 & 1 & $\overline{1}$ \\
\hline$c_{\mathrm{AB}}$ & 1.115 & 1.270 & 1.110 & 1.230 & 1 & 1 & 1 & 1 \\
\hline$(\mathrm{T}) ?$ & no & no & no & $\mathrm{nc}$ & yes & yes & no & no \\
\hline
\end{tabular}

Errors relative to $\operatorname{CCSD}\left(\mathrm{Tc}_{\mathrm{sc}}\right)-\mathrm{F} 12 \mathrm{c} / \mathrm{cc}-\mathrm{pVDZ}-\mathrm{F} 12$. cc-pVDZ-F12 basis set throughout. ${ }^{a} s_{6}=0.225$.

work nearly as well, as does adding a Grimme D2 dispersion correction with $s_{6}=0.225$ (see discussion below together with dRPA75).

At the request of a reviewer, we consider the effect of the new data on the intermolecular separations. In the original S66 paper, the intermolecular separations were obtained by quartic interpolation on the $\{0.90,0.95,1.00,1.05,1.10\} r_{\mathrm{e}}$ data points in S66x8 for each complex. We have repeated this procedure for both the original S66x8 data obtained from www.begdb.com and for the present revised data in Table 1 . The minimumenergy intermolecular separations from both datasets are compared in the master data spreadsheet in the ESI, $\dagger$ while the Cartesian coordinates corresponding to them are also made available in .xyz format in the ESI. $\dagger$
By and large, the higher level of theory in the present data (particularly for the HLC) does not translate into dramatic geometry changes: the separations change by $-0.00074 r_{\mathrm{e}}$ on average (the revised geometries being slightly shorter than the originals), $0.00246 r_{\mathrm{e}}$ mean absolute difference, and $0.00285 r_{\mathrm{e}}$ RMS difference. The largest differences are $+0.0066 r_{\mathrm{e}}$ for benzene $\cdots$ ethene (system 30 ) and $-0.0063 r_{\mathrm{e}}$ for pyridine $\cdots$ ethyne (system 65). Generally speaking, the revised data lead to stretching for the pi stacks and contraction for the hydrogen bonds, with mixed behavior seen for the remaining systems.

How consequential are these geometry differences for the total energy? We evaluated DF-SCS(MI)MP2-F12/cc-pVTZ-F12 energies at both sets of geometries: the RMS difference between the total energies over the S66 systems was found to be just 
Table 12 Error statistics $\left(\mathrm{kcal} \mathrm{mol}^{-1}\right)$ for interaction energies of $\mathrm{S} 66 \times 8$ set calculated with MP2, HF and various DFT methods

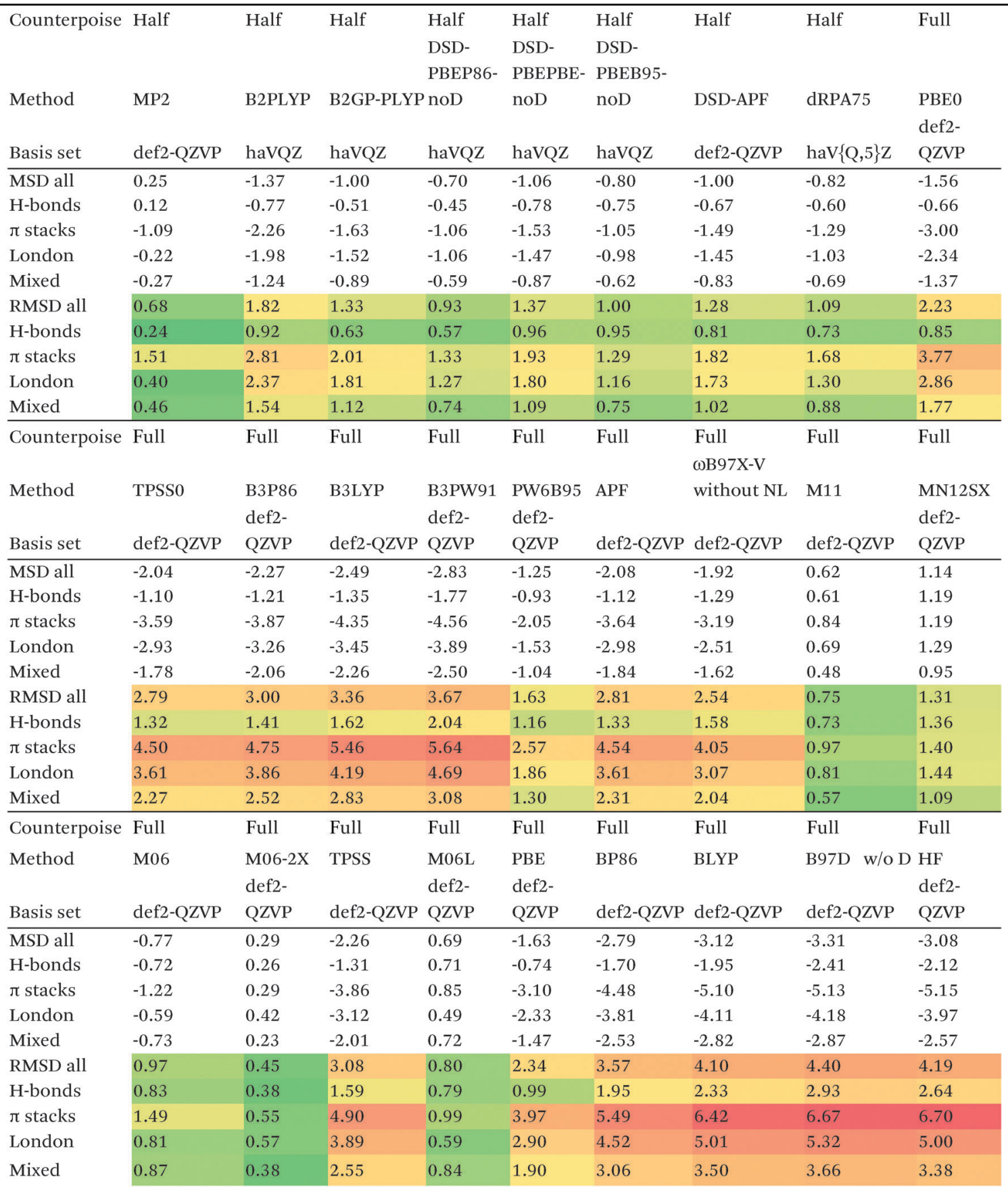

$0.004 \mathrm{kcal} \mathrm{mol}^{-1}$. This is immaterial for all but the highestaccuracy work - and then one would wish to optimize monomer geometries at a higher level as well.

\section{Performance of density functional methods}

Without dispersion corrections. In Table 12, performance for DFT functionals without dispersion correction is considered. Unsurprisingly, the various uncorrected DFT functionals perform quite poorly. Straight HF sets perhaps the low-water-mark for performance, with RMSD from $2.6 \mathrm{kcal} \mathrm{mol}^{-1}$ for $\mathrm{H}$ bonds to
$6.7 \mathrm{kcal} \mathrm{mol}^{-1}$ for $\pi$-stack, and $4.19 \mathrm{kcal} \mathrm{mol}^{-1}$ overall. PBE and PBE0 actually perform a good deal better than that for hydrogen bonds, but still yield a disappointing 2.3 and $2.2 \mathrm{kcal} \mathrm{mol}^{-1}$ overall, respectively, owing primarily to $\pi$ stacks and London complexes.

For straight MP2 this drops to $0.68 \mathrm{kcal} \mathrm{mol}^{-1}$, which would be a lot better if it weren't for the poor performance for $\pi$ stack $\left(\mathrm{RMSD}=1.5 \mathrm{kcal} \mathrm{mol}^{-1}\right)$. MP2 actually holds its own quite well for $\mathrm{H}$-bonds $\left(0.24 \mathrm{kcal} \mathrm{mol}^{-1}\right)$ and reasonably well for London and mixed complexes. 
Table 13 Error statistics $\left(\mathrm{kcal} \mathrm{mol}^{-1}\right)$ for interaction energies of S66 8 set calculated with D2 dispersion corrected MP2, HF and various DFT methods

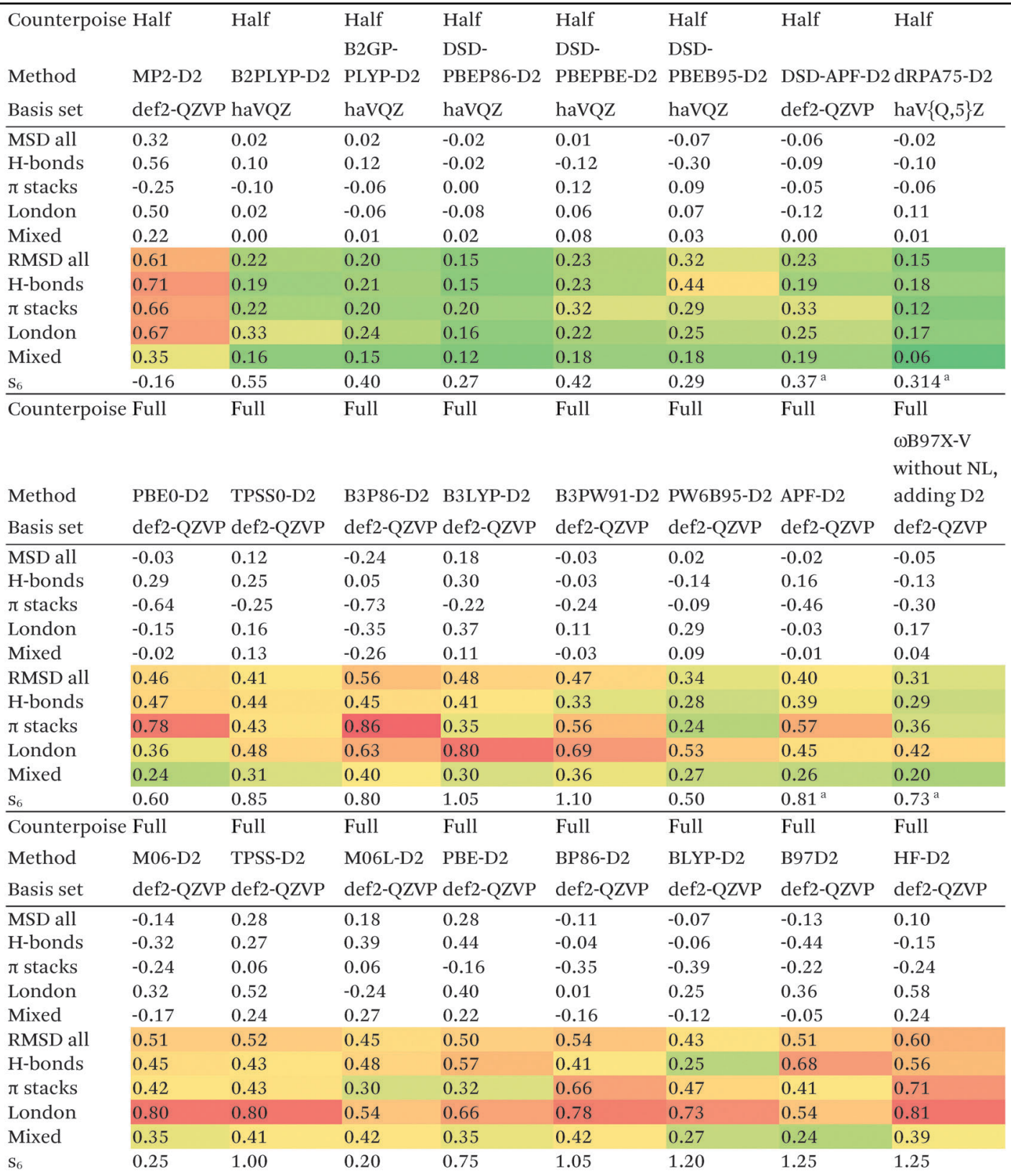

${ }^{a}$ Obtained in the present work by minimization of S66x8 RMSD.

By far the best performer without any dispersion correction is M06-2X, at RMSD $=0.43 \mathrm{kcal} \mathrm{mol}^{-1}$. M06 has over twice that error $\left(0.95 \mathrm{kcal} \mathrm{mol}^{-1}\right)$. At 0.74 and $1.30 \mathrm{kcal} \mathrm{mol}^{-1}$, respectively, M11 and M12 do not offer relief either.

Simple double hybrids perform fairly poorly, RMSD = $1.82 \mathrm{kcal} \mathrm{mol}^{-1}$ for B2PLYP and $1.33 \mathrm{kcal} \mathrm{mol}^{-1}$ for B2GPPLYP. The DSD-noD functionals are in the 0.9-1.4 kcal mol${ }^{-1}$ range, and thus arguably "do not earn their keep", since they are outperformed by straight MP2.

Very recently, Kallay and coworkers proposed ${ }^{81}$ the dRPA75 functional, in which the correlation is obtained from a dRPA (direct random phase approximation, ${ }^{107}$ a.k.a. drCCD or direct ring coupled cluster ${ }^{82,108}$ ) with all doubles calculation in a set of orbitals involving a mixture of $75 \%$ Hartree-Fock-like exchange and 25\% PBE exchange. Using the haVTZ basis set, RMSD without counterpoise correction is a deceptively low $0.37 \mathrm{kcal} \mathrm{mol}^{-1}$; this rises to $0.77 \mathrm{kcal} \mathrm{mol}^{-1}$ for def2-QZVP, $0.71 \mathrm{kcal} \mathrm{mol}^{-1}$ for haVQZ, $0.89 \mathrm{kcal} \mathrm{mol}^{-1}$ for haV5Z, and $1.09 \mathrm{kcal} \mathrm{mol}^{-1}$ at the $\operatorname{haV}\{\mathrm{Q}, 5\} \mathrm{Z}$ extrapolated limit. At the basis set limit, raw and counterpoise-corrected values agree tolerably well, yet one manifestly sees very slow basis set convergence akin to a conventional wavefunction method.

With dispersion corrections. We now consider the simple D2 empirical dispersion. Even this rudimentary correction dramatically improves performance of functionals like PBE, PBE0, and B3LYP; even for straight HF, adding D2 brings down 
RMSD to about $0.6 \mathrm{kcal}^{\mathrm{mol}^{-1}}$ (Table 13). M06, which was designed to implicitly account for intermediate-range dispersion, still benefits from the long-range D2 correction (RMSD lowered from 0.97 to $0.51 \mathrm{kcal} \mathrm{mol}^{-1}$ ) (Table 13). The $\pi$ stacks and/or the London complexes are typically the most problematic subset, but for B97D2 it is actually the hydrogen-bonded complexes. PW6B95D2 puts in a highly creditable performance, at RMSD = $0.33 \mathrm{kcal} \mathrm{mol}^{-1}$, as does the $\omega \mathrm{B} 97 \mathrm{X}-\mathrm{V}$ functional, with the NL correction deleted and replaced by $\mathrm{D} 2$.

Of the simple double hybrids, both B2PLYP-D2 and B2GPPLYP-D2 exhibit excellent performances $(\mathrm{RMSD}=0.22$ and $0.20 \mathrm{kcal} \mathrm{mol}^{-1}$, respectively, even including $\pi$ stack dimers). DSD-PBEP86-D2 yields the best result of all the D2-corrected functionals, at RMSD $=0.15 \mathrm{kcal} \mathrm{mol}^{-1}$.

The most significant improvement is seen for dRPA75. At the half-counterpoise $\operatorname{haV}\{\mathrm{Q}, 5\} \mathrm{Z}$ limit, adding a D2 correction with $s_{6}=0.314$ leads to RMSD $=0.15 \mathrm{kcal} \mathrm{mol}^{-1}$, which changes the performance of dRPA75 from mediocre to best of class. A partial rationale is offered by considering the statistical correlation between $(\mathrm{Tb})$ and the $\mathrm{D} 2$ correction: for the entire S66x8 set, $R^{2}=0.92$. This is perhaps not surprising, from an SAPT perspective, in view of the importance of fourth-order (T) in the correction to the dispersion energy. dRPA can, after all, be seen as an approximation to CCSD. By way of further illustration, we considered adding D2 corrections to $\operatorname{CCSD}\left(\mathrm{F} 12^{*}\right)$ itself, which leads to $s_{6}=0.225$ and RMSD $=0.156 \mathrm{kcal} \mathrm{mol}^{-1}$, not dissimilar to dRPA75-D2.

Switching from D2 to the more sophisticated D3BJ empirical correction improves statistics for virtually all the GGAs, meta-GGAs, and hybrids. (The two exceptions are straight HF - which could be regarded as a "hybrid" with $100 \%$ exact exchange and null correlation - and PW6B95.) For BLYP-D3BJ, for instance, an amazingly low RMSD $=0.23 \mathrm{kcal} \mathrm{mol}^{-1}$ is obtained, while B3LYPD3BJ yields essentially the same performance to within the uncertainty of the reference dataset (Table 14). Several other functionals can attain values around $0.3 \mathrm{kcal} \mathrm{mol}^{-1}$, such as TPSS0-D3BJ and B3PW91-D3BJ.

The APF functional ${ }^{71}\left(\mathrm{RMSD}=0.26 \mathrm{kcal} \mathrm{mol}^{-1}\right.$ for APF-D3BJ) was designed to be more or less “dispersion-free": specifically, it consists of a linear combination of B3PW91 and PBE0 chosen such that the dissociation curve of $\mathrm{Ne}_{2}$ (a paradigmatically dispersion-bound system) is as close to the repulsive HartreeFock results as possible. D3BJ parameters were fitted in this work: noteworthy is the large positive $s_{8}$ coefficient. The latter corresponds to an additional correction in the medium-range region. For perspective, we also fitted D3BJ parameters for straight MP2, and found a large negative $s_{8}=-3.35$, corresponding to RMSD $=0.31 \mathrm{kcal} \mathrm{mol}^{-1}$. For additional perspective, we fitted D3BJ parameters for dRPA75, and found that RMSD dropped to $0.10 \mathrm{kcal} \mathrm{mol}^{-1}$ with both $a_{1}$ and $s_{8}$ constrained to zero. No statistically significant change was seen when relaxing the $s_{8}=0$ constraint. In other words, while dRPA75 does not recover all long-range dispersion, it appears to recover the intermediate range.

Exploring this point a little further, we considered fitting M06-D3BJ. No stable fit could be obtained unless the constraints $a_{1}=s_{8}=0$ were applied, in which case RMSD dropped to $0.40 \mathrm{kcal} \mathrm{mol}^{-1}$. It thus appears that the main benefit of M06 over conventional hybrid GGAs is likewise in the intermediatedistance region (vide supra).

What about the double hybrids? While for B2PLYP-D3BJ, $s_{8}=0.91$ and thus quite nontrivial, for B2GP-PLYP-D3BJ $s_{8}=0.26$ and $a_{1}=0.00$, corresponding to RMSD $=0.23 \mathrm{kcal} \mathrm{mol}^{-1}$. For DSD-PBEP86-D3BJ, on the other hand, a stable fit required setting $s_{8}=a_{1}=0.00$ : this pattern repeats itself across all the DSD double hybrids. Effectively, this means that, for weak interactions, (a) the principal benefit of double hybrids and RPA correlation alike is correction in the intermediate distance region; (b) since straight MP2 overcorrects in that region, this needs to be tempered by either including higher-order correlation (as in dRPA75 or MP2.5) or by throttling the MP2 correlation term (as happens in double hybrids). We attempted replacing the dRPA correlation term in dRPA75 by an MP2-like term, and found qualitatively the same behavior as for ordinary MP2.

Overall, for the double hybrids, improvements from using D3BJ instead of D2 are marginal at best, while DSD-PBEP86-D2 even outperforms DSD-PBEP86-D3BJ. We note that, aside from the $s_{8}$ term being effectively absent (or not fittable at a statistically significant level), a large part of dispersion is already covered by the MP2-like terms.

We optimized a new DSD double hybrid based on the APF "dispersion-free" hybrid, with the idea that this would eliminate some double-counting. It yields a respectable RMSD = $0.23 \mathrm{kcal} \mathrm{mol}^{-1}$, which is however no improvement over DSD-PBEP86-NL.

dRPA75-D3BJ, with $s_{6}=0.3754, a_{2}=4.5048, a_{1}=s_{8}=0$, improves further (to $0.10 \mathrm{kcal} \mathrm{mol}^{-1}$ ) on the already outstanding performance of dRPA75-D2.

In a very recent benchmark study on the conformer energies of the proteinogenic amino acids, we noted excellent performance of the uncorrected dRPA75/def2-QZVP method, RMSD = $0.21 \mathrm{kcal} \mathrm{\textrm {mol } ^ { - 1 }}$ (Boltzmann-weighted, $T_{\mathrm{B}}=1000 \mathrm{~K}$ ) or $0.37 \mathrm{kcal} \mathrm{mol}^{-1}$ (unit weights). We re-evaluated these statistics using dRPA75-D2/def2-QZVP and dRPA75-D3BJ/def2-QZVP. With the D2 dispersion correction, statistics for the conformer set actually were degraded to 0.42 (Boltzmann) and 0.70 (unit weights) kcal $\mathrm{mol}^{-1}$. The D3BJ correction, on the contrary, improved the statistics to 0.14 (Boltzmann) and 0.32 (unit weights) kcal mol ${ }^{-1}$, comparable to the best performers in that paper. It was previously shown (e.g., see ref. 109 and 110 for alkanes) that midrange dispersion interactions are very important in conformer equilibria, and that D2 is often more of a hindrance than a help for these properties owing to the form of the cutoff function; D3BJ, on the other hand, does not exhibit this problem. ${ }^{110}$ As we have shown earlier in the manuscript that unassisted dRPA75 already works well in the medium distance range, adding D2 for conformer equilibria amounts to "fixing what ain't broke".

Concerning range-separated double hybrids, we note that Head-Gordon's recent "survival of the fittest/most transferable" $\omega B 97 \mathrm{X}-\mathrm{V}\left(0.23 \mathrm{kcal} \mathrm{mol}^{-1}\right)$, which involves $\mathrm{NL}$ as its dispersion component, is actually slightly improved by replacing NL with a 
Table 14 Error statistics $\left(\mathrm{kcal} \mathrm{mol}^{-1}\right)$ for interaction energies of S66x8 set calculated with D3BJ dispersion corrected MP2, HF and various DFT methods

\begin{tabular}{|c|c|c|c|c|c|c|c|c|}
\hline Counterpoise & Half & Half & Half & Half & Half & Half & Half & Half \\
\hline $\begin{array}{l}\text { Method } \\
\text { Basis set }\end{array}$ & $\begin{array}{l}\text { MP2-D3BJ } \\
\text { def2-QZVP }\end{array}$ & $\begin{array}{l}\text { B2PLYP-D3BJ } \\
\text { haVQZ }\end{array}$ & $\begin{array}{l}\text { B2GP-PLYP- } \\
\text { D3BJ } \\
\text { haVQZ }\end{array}$ & $\begin{array}{l}\text { DSD-PBEP86- } \\
\text { D3BJ } \\
\text { haVQZ }\end{array}$ & $\begin{array}{l}\text { DSD-PBEPBE- } \\
\text { D3BJ } \\
\text { haVQZ }\end{array}$ & $\begin{array}{l}\text { DSD-PBEB95- } \\
\text { D3BJ } \\
\text { haVQZ }\end{array}$ & $\begin{array}{l}\text { DSD-APF-D3BJ } \\
\text { def2-QZVP }\end{array}$ & $\begin{array}{l}\text { dRPA75-D3B] } \\
\operatorname{haV}\{\mathrm{Q}, 5\} \mathrm{Z}\end{array}$ \\
\hline MSD all & 0.03 & -0.09 & -0.11 & 0.09 & 0.05 & -0.01 & -0.05 & 0.01 \\
\hline H-bonds & 0.20 & -0.02 & -0.01 & 0.04 & -0.13 & -0.29 & -0.09 & -0.02 \\
\hline$\pi$ stacks & -0.17 & -0.03 & -0.08 & 0.27 & 0.36 & 0.32 & 0.08 & -0.04 \\
\hline London & -0.03 & -0.21 & -0.31 & 0.02 & 0.05 & 0.12 & -0.17 & 0.05 \\
\hline Mixed & -0.03 & -0.10 & -0.12 & 0.12 & 0.11 & 0.08 & 0.00 & 0.03 \\
\hline RMSD all & 0.31 & 0.19 & 0.22 & 0.20 & 0.25 & 0.33 & 0.21 & 0.10 \\
\hline H-bonds & 0.34 & 0.13 & 0.13 & 0.12 & 0.23 & 0.43 & 0.18 & 0.09 \\
\hline$\pi$ stacks & 0.45 & 0.19 & 0.16 & 0.38 & 0.47 & 0.43 & 0.33 & 0.15 \\
\hline London & 0.23 & 0.28 & 0.39 & 0.10 & 0.15 & 0.19 & 0.23 & 0.09 \\
\hline Mixed & 0.22 & 0.17 & 0.18 & 0.17 & 0.17 & 0.18 & 0.16 & 0.07 \\
\hline $\mathrm{S}_{6}$ & $1.049^{*}$ & 0.640 & 0.560 & 0.480 & 0.780 & 0.580 & $0.574^{*}$ & $0.375^{*}$ \\
\hline $\mathrm{S}_{8}$ & $-3.351^{*}$ & 0.915 & 0.260 & 0.000 & 0.000 & 0.000 & 0.000 & 0.000 \\
\hline$a_{1}$ & $-0.478^{*}$ & 0.307 & 0.000 & 0.000 & 0.000 & 0.000 & 0.000 & 0.000 \\
\hline $\mathrm{a}_{2}$ & $9.254^{*}$ & 5.057 & 6.333 & 5.600 & 6.100 & 6.200 & $5.623^{*}$ & $4.505^{*}$ \\
\hline Counterpoise & Full & Full & Full & Full & Full & Full & Full & Full \\
\hline $\begin{array}{l}\text { Method } \\
\text { Basis set }\end{array}$ & $\begin{array}{l}\text { PBE0-D3BJ } \\
\text { def2-QZVP }\end{array}$ & $\begin{array}{l}\text { TPSS0-D3BJ } \\
\text { def2-QZVP }\end{array}$ & $\begin{array}{l}\text { B3LYP-D3BJ } \\
\text { def2-QZVP }\end{array}$ & $\begin{array}{l}\text { B3PW91-D3BJ } \\
\text { def2-QZVP }\end{array}$ & $\begin{array}{l}\text { PW6B95-D3BJ } \\
\text { def2-QZVP }\end{array}$ & $\begin{array}{l}\text { APF-D3BJ } \\
\text { def2-QZVP }\end{array}$ & $\begin{array}{l}\omega \mathrm{B} 97 \mathrm{X}-\mathrm{V} \text { without } \\
\mathrm{NL} \text {, adding D3BJ } \\
\text { def2-QZVP }\end{array}$ & $\begin{array}{l}\text { CAM-B3LYP- } \\
\text { D3BJ } \\
\text { def2-QZVP }\end{array}$ \\
\hline MSD all & 0.12 & 0.01 & 0.05 & -0.04 & -0.10 & 0.02 & 0.01 & -0.075 \\
\hline H-bonds & 0.30 & 0.12 & 0.16 & -0.14 & -0.31 & 0.10 & -0.03 & -0.409 \\
\hline$\pi$ stacks & -0.13 & -0.10 & -0.01 & 0.26 & -0.05 & 0.01 & -0.12 & 0.263 \\
\hline London & 0.02 & -0.13 & -0.03 & -0.12 & 0.12 & -0.09 & 0.09 & 0.101 \\
\hline Mixed & 0.11 & 0.03 & -0.01 & -0.04 & -0.03 & 0.02 & 0.09 & 0.025 \\
\hline RMSD all & 0.35 & 0.30 & 0.20 & 0.31 & 0.34 & 0.26 & 0.20 & 0.429 \\
\hline H-bonds & 0.46 & 0.36 & 0.28 & 0.35 & 0.45 & 0.33 & 0.18 & 0.569 \\
\hline$\pi$ stacks & 0.39 & 0.28 & 0.19 & 0.44 & 0.35 & 0.28 & 0.29 & 0.551 \\
\hline London & 0.25 & 0.32 & 0.13 & 0.22 & 0.25 & 0.23 & 0.16 & 0.250 \\
\hline Mixed & 0.21 & 0.20 & 0.13 & 0.20 & 0.23 & 0.18 & 0.18 & 0.204 \\
\hline $\mathrm{S}_{6}$ & 1.000 & 1.000 & 1.000 & 1.000 & 1.000 & $1.000^{*}$ & $1.000^{*}$ & 1.000 \\
\hline $\mathrm{S}_{8}$ & 1.218 & 1.258 & 1.989 & 2.852 & 0.726 & $1.776^{*}$ & $0.126^{*}$ & 2.067 \\
\hline$a_{1}$ & 0.415 & 0.377 & 0.398 & 0.431 & 0.208 & $0.341^{*}$ & $0.211^{*}$ & 0.371 \\
\hline $\mathrm{a}_{2}$ & 4.859 & 4.587 & 4.421 & 4.469 & 6.375 & $4.995^{*}$ & $4.350^{*}$ & 5.474 \\
\hline Counterpoise & Full & Full & Full & Full & Full & Full & Full & Full \\
\hline Method & $\omega \mathrm{B} 97 \mathrm{XD} 3$ & $\begin{array}{l}\text { LC- } \omega \text { PBE- } \\
\text { D3BJ }\end{array}$ & TPSS-D3BJ & PBE-D3BJ & BP86-D3BJ & BLYP-D3BJ & B97D3 & HF-D3BJ \\
\hline Basis set & def2-QZVP & def2-QZVP & def2-QZVP & def2-QZVP & def2-QZVP & def2-QZVP & def2-QZVP & def2-QZVP \\
\hline MSD all & -0.08 & 0.03 & -0.06 & 0.09 & 0.23 & -0.11 & 0.00 & 0.33 \\
\hline H-bonds & 0.02 & 0.18 & -0.03 & 0.28 & 0.11 & -0.14 & -0.28 & 0.54 \\
\hline$\pi$ stacks & 0.02 & -0.14 & -0.10 & -0.22 & 0.67 & 0.05 & 0.24 & -0.22 \\
\hline London & -0.41 & 0.02 & -0.10 & 0.06 & 0.23 & -0.08 & 0.20 & 0.26 \\
\hline Mixed & -0.02 & -0.04 & -0.06 & 0.05 & 0.14 & -0.16 & 0.06 & 0.41 \\
\hline RMSD all & 0.32 & 0.25 & 0.34 & 0.36 & 0.58 & 0.23 & 0.37 & 0.63 \\
\hline H-bonds & 0.16 & 0.30 & 0.29 & 0.39 & 0.48 & 0.26 & 0.47 & 0.80 \\
\hline$\pi$ stacks & 0.34 & 0.26 & 0.46 & 0.55 & 1.07 & 0.25 & 0.41 & 0.59 \\
\hline London & 0.57 & 0.19 & 0.42 & 0.32 & 0.48 & 0.17 & 0.32 & 0.42 \\
\hline Mixed & 0.17 & 0.21 & 0.26 & 0.21 & 0.37 & 0.23 & 0.24 & 0.53 \\
\hline $\mathrm{S}_{6}$ & & 1.000 & 1.000 & 1.000 & 1.000 & 1.000 & 1.000 & 1.000 \\
\hline $\mathrm{S}_{8}$ & & 1.854 & 1.944 & 0.788 & 3.282 & 2.700 & 2.261 & 0.917 \\
\hline$a_{1}$ & & 0.392 & 0.454 & 0.429 & 0.395 & 0.430 & 0.555 & 0.339 \\
\hline$a_{2}$ & & 5.090 & 4.475 & 4.441 & 4.852 & 4.236 & 3.230 & 2.883 \\
\hline
\end{tabular}

D3BJ parameters taken from http://www.thch.uni-bonn.de/tc/downloads/DFT-D3/functionalsbj.html except where indicated by $\left(^{*}\right)$, which were optimized in this work, and for DSD double hybrids, taken from ref. 80.

custom-fitted D3BJ. This may prove useful for codes in which no implementation of NL is available. While CAM-B3LYP-D3BJ does considerably worse than B3LYP-D3BJ, LC- $\omega$ PBE-D3BJ well outperforms both PBE-D3BJ and PBE0-D3BJ. 
Table 15 Error statistics $\left(\mathrm{kcal} \mathrm{mol}^{-1}\right)$ for interaction energies of S66x8 set calculated with MP2, HF and various DFT methods considering NL correction

\begin{tabular}{|c|c|c|c|c|c|c|c|}
\hline Counterpoise & Half & Half & Half & Half & Half & Half & Half \\
\hline Method & B2PLYP-NL & B2GP-PLYP-NL & DSD-PBEP86-NL & DSD-PBEPBE-NL & DSD-PBEB95-NL & DSD-APF-NL & PBE0-NL \\
\hline Basis set & haVQZ & haVQZ & haVQZ & haVQZ & haVQZ & def2-QZVP & def2-QZVP \\
\hline MSD all & -0.01 & 0.01 & 0.02 & -0.01 & -0.06 & -0.03 & 0.03 \\
\hline H-bonds & 0.11 & 0.13 & 0.00 & -0.11 & -0.29 & -0.06 & 0.37 \\
\hline$\pi$ stacks & -0.01 & 0.04 & 0.13 & 0.20 & 0.18 & 0.12 & -0.39 \\
\hline London & -0.12 & -0.12 & -0.05 & -0.03 & 0.06 & -0.11 & -0.18 \\
\hline Mixed & -0.09 & -0.04 & 0.03 & 0.02 & 0.01 & -0.01 & -0.03 \\
\hline RMSD all & 0.18 & 0.17 & 0.12 & 0.18 & 0.27 & 0.16 & 0.45 \\
\hline H-bonds & 0.21 & 0.22 & 0.10 & 0.19 & 0.41 & 0.14 & 0.59 \\
\hline$\pi$ stacks & 0.15 & 0.12 & 0.19 & 0.26 & 0.23 & 0.22 & 0.56 \\
\hline London & 0.19 & 0.19 & 0.15 & 0.19 & 0.14 & 0.20 & 0.32 \\
\hline Mixed & 0.15 & 0.11 & 0.08 & 0.10 & 0.12 & 0.11 & 0.23 \\
\hline $\mathrm{NLb}^{\mathrm{a}}$ & 7.8 & 9.9 & 12.8 & 9.6 & 12.5 & 10.2 & 6.9 \\
\hline Counterpoise & Full & Full & Full & Full & Full & Full & Full \\
\hline Method & TPSS0-NL & B3P86-NL & B3LYP-NL & B3PW91-NL & PW6B95-NL & APF-NL & $\omega \mathrm{B} 97 \mathrm{X}-\mathrm{V}$ \\
\hline Basis set & def2-QZVP & def2-QZVP & def2-QZVP & def2-QZVP & def2-QZVP & def2-QZVP & def2-QZVP \\
\hline MSD all & -0.01 & -0.11 & -0.08 & -0.27 & -0.10 & 0.02 & -0.08 \\
\hline H-bonds & 0.24 & 0.22 & 0.26 & -0.05 & -0.20 & 0.27 & -0.08 \\
\hline$\pi$ stacks & -0.23 & -0.32 & -0.39 & -0.35 & -0.16 & -0.19 & -0.16 \\
\hline London & -0.22 & -0.35 & -0.23 & -0.49 & 0.06 & -0.15 & -0.03 \\
\hline Mixed & -0.07 & -0.24 & -0.22 & -0.35 & -0.07 & -0.05 & -0.07 \\
\hline RMSD all & 0.40 & 0.48 & 0.42 & 0.47 & 0.26 & 0.37 & 0.23 \\
\hline H-bonds & 0.54 & 0.65 & 0.46 & 0.47 & 0.31 & 0.56 & 0.20 \\
\hline$\pi$ stacks & 0.33 & 0.36 & 0.56 & 0.41 & 0.30 & 0.24 & 0.37 \\
\hline London & 0.35 & 0.40 & 0.31 & 0.57 & 0.19 & 0.22 & 0.18 \\
\hline Mixed & 0.24 & 0.33 & 0.33 & 0.43 & 0.22 & 0.20 & 0.19 \\
\hline $\mathrm{NLb}^{\mathrm{a}}$ & 5.5 & 5.3 & 4.8 & 4.5 & 9.0 & 5.4 & 6.0 \\
\hline NLc & & & & & & & 0.01 \\
\hline Counterpoise & Full & Full & Full & Full & Full & Full & Full \\
\hline Method & M06-NL & TPSS-NL & PBE-NL & BP86-NL & BLYP-NL & B97D-NL & HF-NL \\
\hline Basis set & def2-QZVP & def2-QZVP & def2-QZVP & def2-QZVP & def2-QZVP & def2-QZVP & def2-QZVP \\
\hline MSD all & -0.16 & 0.01 & 0.12 & -0.10 & -0.14 & -0.07 & -0.14 \\
\hline H-bonds & -0.33 & 0.22 & 0.41 & 0.12 & 0.09 & -0.18 & -0.17 \\
\hline$\pi$ stacks & -0.22 & -0.13 & -0.24 & -0.10 & -0.28 & 0.16 & -0.20 \\
\hline London & 0.28 & -0.09 & 0.06 & -0.22 & -0.18 & 0.08 & -0.11 \\
\hline Mixed & -0.21 & -0.09 & 0.01 & -0.27 & -0.31 & -0.14 & -0.08 \\
\hline RMSD all & 0.44 & 0.34 & 0.41 & 0.44 & 0.33 & 0.33 & 0.56 \\
\hline H-bonds & 0.44 & 0.45 & 0.55 & 0.58 & 0.26 & 0.32 & 0.51 \\
\hline$\pi$ stacks & 0.35 & 0.29 & 0.47 & 0.32 & 0.38 & 0.36 & 0.91 \\
\hline London & 0.59 & 0.29 & 0.27 & 0.31 & 0.24 & 0.36 & 0.46 \\
\hline Mixed & 0.34 & 0.24 & 0.22 & 0.35 & 0.40 & 0.31 & 0.42 \\
\hline$N_{L} b^{a}$ & 14.4 & 5.0 & 6.4 & 4.4 & 4.0 & 3.6 & 3.9 \\
\hline
\end{tabular}

${ }^{a}$ NLb parameters obtained in ref. 91 and taken from Table 1.Any remaining NLb parameters taken from ref. 90, except $\omega B 97 \mathrm{X}-\mathrm{V}$ from the original ref. 76.

We now turn to the Vydrov-Van Voorhis ${ }^{89}$ "nonlocal" (NL) dispersion correction. This correction effectively only has a single adjustable parameter NLb, which governs short-range attenuation. There are no atomic parameters, as this information is extracted $a$ posteriori from the calculated electron density.

For many functionals such as PBE, PBE0, TPSS0, BLYP, B3LYP, and B3PW91, NL actually performs worse than D3BJ, while for TPSS the two appear to be of the same quality. For
BP86, B97D, HF, and PW6B95, it clearly is superior to D3BJ, besides having the obvious advantage that no atomic parameters are required at all (Table 15).

Among the simple double hybrids, NL does marginally better than D2 and D3BJ for B2GP-PLYP (RMSD $=0.17 \mathrm{kcal} \mathrm{mol}^{-1}$ ).

$\mathrm{Yu}^{16}$ proposed adding the correction to spin-component scaled double hybrids. His optimization procedure neglects the quite considerable BSSE in the S66 series: in the framework of our recent 
study on amino acid conformers ${ }^{91}$ we reoptimized his proposed restriction parameters: these are the ones used in the present work.

In relative terms, it may well be the DSD double hybrids that benefit most. For DSD-PBEP86-NL, RMSD drops to just $0.12 \mathrm{kcal} \mathrm{mol}^{-1}$, coupled with the excellent performance of DSD-PBEP86 for thermochemical and kinetic properties ${ }^{79,80}$ as well as for vibrational frequencies. ${ }^{111}$ But also for DSDPBEPBE-NL (0.18 kcal mol $\left.{ }^{-1}\right)$, DSD-APF-NL (0.16 kcal mol $\left.{ }^{-1}\right)$, and to a lesser extent for DSD-PBEhB95-NL $\left(0.27 \mathrm{kcal} \mathrm{mol}^{-1}\right)$ significant improvements over the corresponding D3BJ functionals were seen. The $\pi$-stacking complexes benefit most, their RMS errors typically being about halved.

The DSD-APF-NL functional, which yielded the second best performance, was actually created by simply applying $0.411^{*} \mathrm{DSD}$ $\mathrm{PBE}+(1-0.411) *$ DSD-BPW91, each with parameters taken from ref. 80

Functionals of the form DSD-XC-NL not only appear to have excellent performance, but they come with two additional advantages: (1) there is no need for an elaborate set of atomic dispersion parameters; (2) the method is no longer open to the criticism that it involves "adulterating" an electronic structure method with Lennard-Jones type corrections.

The advantages of NL for double hybrids are not evident if only equilibrium values (i.e., S66) are considered: it is at compressed geometries that they yield their greatest benefits.

The original D3BJ parameters for the DSD functionals were optimized using half-counterpoise with the relatively small def2-TZVP basis set, mostly from the S22 and Grubbs catalyst benchmarks. We considered performance when refitting against the present S66x8 data. For DSD-PBEP86-D3BJrefit, we obtain RMSD = $0.158 \mathrm{kcal} \mathrm{mol}^{-1}$ for $s_{6}=0.468$ and $a_{2}=5.857$, which closes some of the gap with DSD-PBEP86-NL but not all of it. For DSD-PBEPBED3BJ, RMSD $=0.193 \mathrm{kcal} \mathrm{mol}^{-1}$ is statistically indistinguishable from the DSD-PBEPBE-NL number; the revised parameters are $s_{6}=0.518$ and $a_{2}=4.846$. For DSD-PBEhB95-D3BJ, refitting yields $s_{6}=0.263$ and a somewhat anomalous $a_{2}=3.360$, with RMSD $=$ $0.204 \mathrm{kcal} \mathrm{mol}^{-1}$ actually better than DSD-PBEhB95-NL.

How does the substitution of NL for D3BJ affect performance for other test sets, such as barrier heights, atomization energies, and the like? We considered the six training sets used in parametrizing the original functionals. The results can briefly be summarized as implying that D3BJ and NL yield results of similar quality considering the residual uncertainty in the reference values. It therefore appears to pass the "above all, do no harm" test.

On the other hand, D3BJ is available in several additional codes beyond ORCA, and derivatives are trivial to implement (unlike for NL).

\section{SAPT results and a proposed new noncovalent character index}

From a SAPT perspective, the SCF component of the interaction energy can be decomposed as follows: ${ }^{39,112}$

$\mathrm{IE}_{\mathrm{SCF}}=E_{\mathrm{elst}}^{(10)}+E_{\mathrm{exch}}^{(10)}+E_{\text {ind }}^{(20)}+E_{\text {exch-ind }}^{(20)}+E_{\text {ind }}^{(30)}+E_{\text {exch-ind }}^{(30)}+\delta \mathrm{SCF}_{3}$

$$
\mathrm{IE}_{\mathbf{S C F}}=E_{\text {elst }}^{(10)}+E_{\text {exch }}^{(10)}+E_{\text {ind }}^{(20)}+E_{\text {exch-ind }}^{(20)}+\delta \mathrm{SCF}_{2}
$$

where in this and the following equations, blue terms are attractive, red terms are repulsive, black terms can go either way, the two superscripts indicate order of inter-and intramolecular perturbation theory, respectively, and the subscript "elst", "ind”, “exch" stand for electrostatic, induction, and exchange, respectively. $\delta \mathrm{SCF}_{3}$ is a catchall term for any remaining higher-order electrostatic, induction, and exchange terms. "exch-ind" stands for the exchange correction to induction.

The second-order component can be decomposed as: $:^{39,112}$

$$
\begin{aligned}
\mathrm{IE}_{\mathrm{MP} 2}-\mathrm{IE}_{\mathrm{SCF}}= & E_{\text {disp }}^{(20)}+E_{\text {exch-disp }}^{(20)}+E_{\text {elst }}^{(12)}+E_{\text {exch-elst }}^{(12)}+E_{\text {ind }}^{(22)} \\
& +E_{\text {exch-ind }}^{(22)}+\delta \mathrm{MP} 2
\end{aligned}
$$

where "disp" stands for dispersion, "exch-disp" for the exchange correction to dispersion, and $\delta \mathrm{MP} 2$ is a catchall term for higher-order electrostatic and induction terms. It is important to realize that $E_{\text {disp }}^{(20)}$ has identical $\alpha \alpha$ and $\alpha \beta$ components: inclusion of $E_{\text {exch-disp }}^{(20)}$ introduces spin dependence.

For the third-order terms, eqn (6:35) of Chalasinski and Szczesniak $^{102}$ implies that

$\mathrm{IE}_{\mathrm{MP} 3}-\mathrm{IE}_{\mathrm{MP2}}=E_{\mathrm{disp}}^{(21)}+E_{\text {exch-disp }}^{(21)}+E_{\mathrm{elst}}^{(13)}+E_{\text {disp }}^{(30)}+E_{\text {exch-disp }}^{(30)}+\delta \mathrm{MP} 3$

Additional terms will appear at fourth order:

$$
\mathrm{IE}_{\mathrm{MP} 4}-\mathrm{IE}_{\mathrm{MP} 3}=E_{\mathrm{disp}}^{(22)}(\mathrm{SDQ})+E_{\mathrm{disp}}^{(22)}(\mathrm{T})+\ldots
$$

At the two least expensive levels of SAPT, SAPT0 and SAPT2, the interaction energy can be decomposed as:

$$
\mathbf{I E}_{\mathrm{SAPTO}}=E_{\text {elst }}^{(10)}+E_{\text {exch }}^{(10)}+E_{\text {ind }}^{(20)}+E_{\text {exch-ind }}^{(20)}+E_{\text {disp }}^{(20)}+E_{\text {exch-disp }}^{(20)}
$$

$$
\mathrm{IE}_{\mathrm{SAPT} 2}=\mathrm{IE}_{\mathrm{SAPT0}}+E_{\mathrm{elst}}^{(12)}+E_{\text {exch-elst }}^{(12)}+E_{\text {ind }}^{(22)}+E_{\text {exch-ind }}^{(22)}
$$

Let us now consider the variable:

$$
\begin{aligned}
\mathrm{NDF} 2 & =\frac{E_{\mathrm{disp}}^{(2)}}{\mathrm{IE}^{(2)}}-1=\frac{E_{\mathrm{disp}}^{(20)}+E_{\mathrm{exch}-\mathrm{disp}}^{(20)}}{\mathrm{IE}_{\mathrm{ss}}^{(2)}+\mathrm{IE}_{\mathrm{ab}}^{(2)}}-1 \\
& =\frac{E_{\mathrm{disp}}^{(20)}+E_{\mathrm{exch}-\mathrm{disp}}^{(20)}}{\mathrm{IE}_{\mathrm{aa}}^{(2)}+\mathrm{IE}_{\mathrm{bb}}^{(2)}+\mathrm{IE}_{\mathrm{ab}}^{(2)}}-1
\end{aligned}
$$

where $\mathrm{IE}^{(2)}$ stands for the MP2 correlation component to the interaction energy, and $\mathrm{IE}_{\mathrm{ss}}^{(2)}$ and $\mathrm{IE}_{\mathrm{ab}}^{(2)}$ stand for the same-spin and opposite-spin components thereof, respectively. In a system dominated by dispersion, NDF2 (non-dispersion fraction at 2nd order) will be close to zero, while in a system with significant nondispersion contributions to the 2nd-order correlation energy, it will typically be positive (correlation corrections to the electrostatic interaction energy tend to be repulsive). 
NDF2 can be computed from an MP2 and an SAPT0 calculation in the same basis set. However, let us now consider the following variable that just requires an MP2 or RI-MP2 calculation:

$$
\mathrm{CSPI}=\frac{\mathrm{IE}_{\mathrm{ss}}^{(2)}-\mathrm{IE}_{\mathrm{ab}}^{(2)}}{\mathrm{IE}_{\mathrm{ss}}^{(2)}+\mathrm{IE}_{\mathrm{ab}}^{(2)}}
$$

Over the S66x8 set, with the haVTZ basis set, we found that NDF2 and CSPI (correlation spin polarization index) have a squared correlation coefficient of no less than 0.991 (see Table S1 in ESI $\dagger$ ). We may therefore safely conclude that they contain the same chemical information.

For a system where the interaction energy is dominated by $E_{\text {disp }}^{(20)}$, CSPI will approach zero; in systems where non-dispersion factors play a role in the correlation part of the interaction energy, CSPI will depart from zero. However, for highly stretched systems, absolute values of the same-spin and opposite component may be so small that CSPI suddenly flips sign. In order to avoid this problem, we will instead consider

$$
\mathrm{DEBC}=\sqrt{\frac{\mathrm{CSPI}^{2}}{1+\mathrm{CSPI}^{2}}}=\sqrt{\frac{1}{2}-\frac{\mathrm{IE}_{\mathrm{ss}}^{(2)} \mathrm{IE}_{\mathrm{ab}}^{(2)}}{\left(\mathrm{IE}_{\mathrm{ss}}^{(2)}\right)^{2}+\left(\mathrm{IE}_{\mathrm{ab}}^{(2)}\right)^{2}}}
$$

where the acronym stands for dispersion-electrostatic balance in correlation. In the long-distance limit for, e.g., hydrogenbonded complexes, the rapidly decaying dispersive component will be small compared to the more slowly decaying nondispersion terms (which are typically repulsive in the correlation component), and as a result the second term will strive to $-\frac{1}{2}$ and DEBC will approach unity. DEBC thus moves on a scale from 0 for purely dispersive (e.g., argon dimer) to 1 for purely nondispersive.

However, at very long distance in systems dominated by nondispersion effects (e.g., acetic acid dimer), $\mathrm{IE}^{(2)}$ will be negligible compared to $\mathrm{IE}_{\mathrm{SCF}}$ and hence DEBC may not be very informative anymore. We will instead consider two additional indices. One is the fraction of the interaction energy accounted for at the Hartree-Fock level

$$
\% \mathrm{HF}=\frac{(100 \%) \times \mathrm{IE}_{\mathrm{SCF}}}{\mathrm{IE}_{\mathrm{SCF}}+\mathrm{IE}_{\mathrm{ss}}^{(2)}+\mathrm{IE}_{\mathrm{ab}}^{(2)}+\mathrm{IE}_{\mathrm{HLC}}} \approx \frac{(100 \%) \times \mathrm{IE}_{\mathrm{SCF}}}{\mathrm{IE}_{\mathrm{SCF}}+\mathrm{IE}_{\mathrm{ss}}^{(2)}+\mathrm{IE}_{\mathrm{ab}}^{(2)}}
$$

For systems dominated by electrostatic effects (e.g., $\mathrm{H}_{2} \mathrm{O}$ dimer at long distance), $\% \mathrm{HF}$ will approach $100 \%$; for systems where the primary HF-level component is exchange repulsion (e.g. alkane dimers), \%HF will be negative.

A second index is the fraction of the interaction energy accounted for by post-MP2 correlation effects:

$$
\% \mathrm{HLC}=\frac{(100 \%) \times \mathrm{IE}_{\mathrm{HLC}}}{\mathrm{IE}_{\mathrm{SCF}}+\mathrm{IE}_{\mathrm{ss}}^{(2)}+\mathrm{IE}_{\mathrm{ab}}^{(2)}+\mathrm{IE}_{\mathrm{HLC}}}
$$

This index will typically be low for systems dominated by electrostatic effects. For systems dominated by London dispersion (e.g., alkane dimers), it is empirically found to be small as well, since an error compensation appears to take place between neglect of (attractive) connected triple excitations and neglect of (typically repulsive) third- and fourth-order double excitations. In the S66x8 set, large values of \%HLC are seen for $\pi$-stacking interactions.

In short, by consideration of three indices derived from the calculated interaction energy, one can infer the dominant interaction type in a system even without resorting to SAPT calculations.

A complete tabulation, complete with the Hobza disp/elec ratio $^{9}$ of values for all the S66x8 systems is given in the ESI. $\dagger$ In Table 16, we present data for some representative systems.

First, let us consider the acetic acid dimer with its strong double hydrogen bond. The Hobza ratio is solidly in the electrostatic range. CSPI and therefore DEBC are large, \%HLC is close to zero, and as the dimer is pulled apart, the \%SCF in the interaction energy approach is $100 \%$. At long distance, the interaction energy indeed behaves similar to the $R^{-3}$ power law expected for dipole-dipole electrostatic interactions.

Next, the stacked benzene dimer. Here, we find a small negative CSPI and a large HLC fraction. The HF fraction is negative throughout, consisting effectively of exchange repulsion. Long-range behavior is in fact not dissimilar to the $R^{-5}$ expected for a quadrupole-quadrupole interaction.

For the stacked uracil dimer, we see something similar at short range, but at longer distances we see HLC becoming fairly unimportant, CSPI rising, and HF capturing an increasing positive fraction of the interaction energy. This reflects that, unlike the benzene dimer, there is a dipole-dipole interaction at longer distances in the uracil dimer.

For pentane dimer, CSPI stays small throughout, as does the \%HLC. The HF contribution is repulsive but tapers off quickly at long distances, where the behavior is dominated by the London interaction.

In the mixed-influence benzene-water complex, on the other hand, CSPI is near zero at short distances but goes up at long distances (where a dipole-quadrupole interaction dominates), while the HLC fraction is substantial at short distances but tapers off to near zero at longer ones, and the HF fraction is small at short distances but approaches unity in the long-distance regime.

\section{Conclusions}

We have presented a revision of the $566 \times 8$ dataset by means of explicitly correlated methods, combining basis set limit MP2-F12 energies with CCSD( $\left.\mathrm{Tc}_{\mathrm{sc}}\right)$-F12b/cc-pVDZ-F12 high level corrections. Based on assessments for smaller datasets, we deem our results reliable to about $0.05 \mathrm{kcal} \mathrm{mol}^{-1} \mathrm{RMS}$. The RMS deviation from the original $566 \times 8$ reference data is $0.11 \mathrm{kcal} \mathrm{mol}^{-1}$, comparable to the performance of the best DFT levels considered here.

Most levels of DFT perform quite poorly in the absence of dispersion corrections: somewhat surprisingly, that is even the case for the double hybrids and for dRPA75. Even the simple D2 empirical dispersion leads to substantial improvement, especially for dRPA75-D2 $\left(s_{6}=0.31, \mathrm{RMSD}=0.13 \mathrm{kcal} \mathrm{mol}^{-1}\right)$ 
Table 16 Indices for type of noncovalent interaction, and their evolution along the dissociation curve, for selected systems in the S66 set. Interaction energies in $\mathrm{kcal} \mathrm{mol}^{-1}$ added for clarity

\begin{tabular}{|c|c|c|c|c|c|c|c|}
\hline & NDF2 & CSPI & DEBC & $\begin{array}{l}\text { Hobza ratio } \\
\text { disp/(elec + ind })\end{array}$ & $\% \mathrm{HF}$ & $\% \mathrm{HLC}$ & IE $\left(\mathrm{kcal} \mathrm{mol}^{-1}\right)$ \\
\hline \multicolumn{8}{|c|}{ H-bond $\cdots\left(\mathrm{CH}_{3} \mathrm{COOH}\right)_{2}$} \\
\hline $20 \cdots 0.90 r_{\mathrm{e}}$ & 2.656 & 1.046 & 0.723 & 0.166 & 80.5 & -2.0 & -17.970 \\
\hline $20 \cdots 0.95 r_{\mathrm{e}}$ & 2.515 & 1.037 & 0.720 & 0.170 & 85.0 & -2.2 & -19.228 \\
\hline $20 \cdots 1.00 r_{\mathrm{e}}$ & 2.440 & 1.045 & 0.723 & 0.173 & 87.9 & -2.4 & -19.469 \\
\hline $20 \cdots 1.05 r_{\mathrm{e}}$ & 2.417 & 1.068 & 0.730 & 0.176 & 90.1 & -2.6 & -19.049 \\
\hline $20 \cdots 1.10 r_{\mathrm{e}}$ & 2.439 & 1.105 & 0.742 & 0.178 & 91.8 & -2.8 & -18.219 \\
\hline $20 \cdots 1.25 r_{\mathrm{e}}$ & 2.760 & 1.322 & 0.798 & 0.180 & 95.4 & -3.1 & -14.736 \\
\hline $20 \cdots 1.50 r_{\mathrm{e}}$ & 4.841 & 2.467 & 0.927 & 0.167 & 99.3 & -3.2 & -9.289 \\
\hline $20 \cdots 2.00 r_{\mathrm{e}}$ & -6.091 & -3.304 & 0.957 & 0.126 & 105.1 & -2.9 & -3.611 \\
\hline \multicolumn{8}{|c|}{$\pi$-stack $\left(\mathrm{C}_{6} \mathrm{H}_{6}\right)_{2}$ parallel displaced } \\
\hline $24 \cdots 0.90 r_{\mathrm{e}}$ & 0.028 & -0.067 & 0.067 & 1.535 & -185.2 & 53.8 & -0.105 \\
\hline $24 \cdots 0.95 r_{\mathrm{e}}$ & 0.020 & -0.061 & 0.061 & 2.062 & -111.4 & 39.5 & -2.016 \\
\hline $24 \cdots 1.00 r_{\mathrm{e}}$ & 0.014 & -0.056 & 0.056 & 2.869 & -75.9 & 32.5 & -2.725 \\
\hline $24 \cdots 1.05 r_{\mathrm{e}}$ & 0.008 & -0.052 & 0.051 & 4.229 & -55.9 & 28.5 & -2.813 \\
\hline $24 \cdots 1.10 r_{\mathrm{e}}$ & 0.003 & -0.048 & 0.048 & 6.930 & -43.7 & 26.1 & -2.607 \\
\hline $24 \cdots 1.25 r_{\mathrm{e}}$ & -0.014 & -0.042 & 0.042 & -18.003 & -28.1 & 22.7 & -1.578 \\
\hline $24 \cdots 1.50 r_{\mathrm{e}}$ & -0.043 & -0.044 & 0.044 & -4.045 & -29.5 & 21.7 & -0.515 \\
\hline $24 \cdots 2.00 r_{\mathrm{e}}$ & -0.083 & -0.064 & 0.064 & -2.242 & -53.0 & 23.1 & -0.072 \\
\hline \multicolumn{8}{|c|}{$\pi$-stack (uracil) $)_{2}$ stacked } \\
\hline $26 \cdots 0.90 r_{\mathrm{e}}$ & 0.174 & 0.045 & 0.045 & 0.963 & -68.1 & 18.4 & -7.976 \\
\hline $26 \cdots 0.95 r_{\mathrm{e}}$ & 0.167 & 0.054 & 0.054 & 1.069 & -23.4 & 12.8 & -9.640 \\
\hline $26 \cdots 1.00 r_{\mathrm{e}}$ & 0.164 & 0.064 & 0.064 & 1.157 & -0.4 & 9.8 & -9.976 \\
\hline $26 \cdots 1.05 r_{\mathrm{e}}$ & 0.164 & 0.075 & 0.074 & 1.220 & 13.7 & 8.0 & -9.590 \\
\hline $26 \cdots 1.10 r_{\mathrm{e}}$ & 0.166 & 0.086 & 0.086 & 1.255 & 23.2 & 6.7 & -8.848 \\
\hline $26 \cdots 1.25 r_{\mathrm{e}}$ & 0.193 & 0.128 & 0.127 & 1.209 & 40.1 & 4.4 & -6.217 \\
\hline $26 \cdots 1.50 r_{\mathrm{e}}$ & 0.301 & 0.227 & 0.222 & 0.927 & 55.7 & 2.5 & -3.196 \\
\hline $26 \cdots 2.00 r_{\mathrm{e}}$ & 0.840 & 0.647 & 0.543 & 0.521 & 77.9 & -0.4 & -1.034 \\
\hline \multicolumn{8}{|c|}{ London (n-pentane $)_{2}$} \\
\hline $34 \cdots 0.90 r_{\mathrm{e}}$ & 0.079 & -0.015 & 0.015 & 2.216 & -268.8 & 11.3 & -2.919 \\
\hline $34 \cdots 0.95 r_{\mathrm{e}}$ & 0.064 & -0.014 & 0.014 & 2.804 & -138.9 & 5.9 & -3.674 \\
\hline $34 \cdots 1.00 r_{\mathrm{e}}$ & 0.053 & -0.013 & 0.013 & 3.575 & -85.8 & 3.8 & -3.820 \\
\hline $34 \cdots 1.05 r_{\mathrm{e}}$ & 0.043 & -0.012 & 0.012 & 4.601 & -57.4 & 2.7 & -3.651 \\
\hline $34 \cdots 1.10 r_{\mathrm{e}}$ & 0.034 & -0.011 & 0.011 & 5.982 & -40.3 & 2.1 & -3.337 \\
\hline $34 \cdots 1.25 r_{\mathrm{e}}$ & 0.017 & -0.008 & 0.008 & 14.297 & -16.0 & 1.7 & -2.257 \\
\hline $34 \cdots 1.50 r_{\mathrm{e}}$ & 0.003 & -0.005 & 0.005 & 185.803 & -4.7 & 2.2 & -1.066 \\
\hline $34 \cdots 2.00 r_{\mathrm{e}}$ & -0.003 & -0.003 & 0.003 & -42.381 & -2.6 & 3.5 & -0.278 \\
\hline \multicolumn{8}{|c|}{$\pi$-stack plus dipole-quadrupole: $\left(\mathrm{C}_{6} \mathrm{H}_{6}\right)_{2}$ T-shaped } \\
\hline $47 \cdots 0.90 r_{\mathrm{e}}$ & 0.082 & -0.018 & 0.018 & 1.470 & -135.8 & 35.8 & -1.657 \\
\hline $47 \cdots 0.95 r_{\mathrm{e}}$ & 0.076 & -0.010 & 0.010 & 1.735 & -70.7 & 25.3 & -2.603 \\
\hline $47 \cdots 1.00 r_{\mathrm{e}}$ & 0.072 & -0.004 & 0.004 & 2.014 & -39.0 & 20.3 & -2.898 \\
\hline $47 \cdots 1.05 r_{\mathrm{e}}$ & 0.070 & 0.003 & 0.003 & 2.290 & -20.4 & 17.3 & -2.853 \\
\hline $47 \cdots 1.10 r_{\mathrm{e}}$ & 0.070 & 0.009 & 0.009 & 2.539 & -8.3 & 15.5 & -2.643 \\
\hline $47 \cdots 1.25 r_{\mathrm{e}}$ & 0.072 & 0.027 & 0.027 & 2.955 & 10.7 & 12.8 & -1.804 \\
\hline $47 \cdots 1.50 r_{\mathrm{e}}$ & 0.088 & 0.052 & 0.052 & 2.668 & 23.2 & 11.4 & -0.856 \\
\hline $47 \cdots 2.00 r_{\mathrm{e}}$ & 0.136 & 0.095 & 0.095 & 1.783 & 34.6 & 9.9 & -0.237 \\
\hline \multicolumn{8}{|c|}{ Mixed-influence: $\left(\mathrm{C}_{6} \mathrm{H}_{6}\right) \cdots \mathrm{H}_{2} \mathrm{O}$} \\
\hline $54 \cdots 0.90 r_{\mathrm{e}}$ & 0.194 & 0.048 & 0.048 & 0.732 & -30.3 & 14.9 & -2.766 \\
\hline $54 \cdots 0.95 r_{\mathrm{e}}$ & 0.206 & 0.063 & 0.063 & 0.739 & 5.9 & 10.5 & -3.186 \\
\hline $54 \cdots 1.00 r_{\mathrm{e}}$ & 0.221 & 0.080 & 0.080 & 0.731 & 25.5 & 8.2 & -3.250 \\
\hline $54 \cdots 1.05 r_{\mathrm{e}}$ & 0.241 & 0.099 & 0.099 & 0.711 & 38.0 & 6.8 & -3.121 \\
\hline $54 \cdots 1.10 r_{\mathrm{e}}$ & 0.266 & 0.121 & 0.120 & 0.680 & 46.9 & 5.9 & -2.896 \\
\hline $54 \cdots 1.25 r_{\mathrm{e}}$ & 0.374 & 0.203 & 0.199 & 0.558 & 63.3 & 4.5 & -2.105 \\
\hline $54 \cdots 1.50 r_{\mathrm{e}}$ & 0.717 & 0.439 & 0.402 & 0.375 & 78.0 & 3.7 & -1.160 \\
\hline $54 \cdots 2.00 r_{\mathrm{e}}$ & 4.515 & 2.948 & 0.947 & 0.193 & 92.7 & 3.2 & -0.419 \\
\hline
\end{tabular}

and for DSD-PBEP86-D2 $\left(s_{6}=0.27\right.$, RMSD $\left.=0.15 \mathrm{kcal} \mathrm{mol}^{-1}\right)$. Below the fifth rung, $\omega \mathrm{B} 97 \mathrm{X}-\mathrm{V}$ without NL, adding $\mathrm{D} 2$ is seen as the best performer $\left(s_{6}=0.73\right.$, RMSD $\left.=0.31 \mathrm{kcal} \mathrm{mol}^{-1}\right)$.

The D3BJ correction leads to further improvements for GGAs and hybrids, much less so for fifth-rung functionals. (D3BJ parameters for a number of additional functionals were optimized in this work.) Significantly, the optimized $s_{8}$ coefficient for the $R^{-8}$ term is close to zero for the double hybrids and dRPA75, or needs to be fixed at zero to get a stable fit. In contrast, for the APF functional (which is constructed to be dispersion-free on average), $s_{6}=1.776$, while for MP2, $s_{6}=-3.351$. This illustrates that the primary benefit of fifth-rung functionals for noncovalent 
interactions lies in the handling of medium-range interactions: in straight MP2, overcorrection takes place in that region, which is remedied in the case of dRPA75-D3BJ by higher-order correlation corrections, and in the double hybrids by the use of a mixture of GGA and KS-MP2 correlation. dRPA75-D3BJ actually falls below the RMSD $=0.10 \mathrm{kcal} \mathrm{mol}^{-1}$ threshold. B3LYP-D3BJ performs surprisingly well at RMSD $=0.20 \mathrm{kcal} \mathrm{mol}^{-1}$.

Considering S66x8 in tandem with the amino acid conformers illustrates why it is worthwhile to consider multiple benchmarks for evaluation lower-level methods: dRPA75-D3BJ performs well on both sets, dRPA75 and dRPA75-D2 each on only one set.

A nonlocal (Vydrov-Van Voorhis 2010, or VV10) correlation model performs less well than D3BJ for some GGAs and hybrids, while it is superior for the double hybrids, particularly for DSD-PBEP86-NL, with RMSD $=0.12 \mathrm{kcal} \mathrm{mol}^{-1}$. Among the range-separated hybrids, $\omega \mathrm{B} 97 \mathrm{X}-\mathrm{V}$ stands out, with RMSD = $0.23 \mathrm{kcal} \mathrm{mol}^{-1}$.

A caveat is due here: the benchmark study in the present paper only concerns noncovalent interactions and any conclusions reached about the performance of specific DFT methods are not necessarily applicable to other properties. It is however worth mentioning that one of the best performers for the S66x8 benchmark, namely the DSD-PBEP86-D3BJ double hybrid, ${ }^{79,80}$ also was found to yield outstanding performance for general thermochemistry $^{79,80}$ and reaction barrier heights, ${ }^{79,80}$ as well as for vibrational frequencies. ${ }^{111}$ The same remarks apply, to a lesser extent, for the B2GP-PLYP-D2 double hybrid. ${ }^{78}$ The computational surcharge for such approaches is actually fairly modest if the RI-MP2 (resolution of the identity MP2) method $^{32,33}$ can be used for the MP2-like step.

Informative as SAPT may be about the character of a given interaction, a collection of three energetically based indices offer similar information. Two of those are the percentages of Hartree-Fock and of post-MP2 correlation effects in the interaction energy: the third

$$
\mathrm{DEBC}=\sqrt{\frac{1}{2}-\frac{\mathrm{IE}_{\mathrm{ss}}^{(2)} \mathrm{IE}_{\mathrm{ab}}^{(2)}}{\left(\mathrm{IE}_{\mathrm{ss}}^{(2)}\right)^{2}+\left(\mathrm{IE}_{\mathrm{ab}}^{(2)}\right)^{2}}}
$$

describes the character of the MP2 correlation contribution, ranging from 0 (purely dispersion) to 1 (purely other effects).

In the context of $\operatorname{CCSD}(\mathrm{T})-\mathrm{F} 12 \mathrm{~b}$ calculations, we propose (Tb), namely an improved, parameter-free scaling for the (T) contribution based on the $E_{\text {corr }}[\mathrm{CCSD}-\mathrm{F} 12 \mathrm{~b}] / E_{\text {corr }}[\mathrm{CCSD}]$ rather than the $E_{\text {corr }}[\mathrm{MP} 2-\mathrm{F} 12] / E_{\text {corr }}[\mathrm{MP} 2]$ ratio. Similarly, we propose (Tc) for $\operatorname{CCSD}\left(\mathrm{F} 12^{*}\right)(\mathrm{T})$ calculations, where the scaling is done by the $E_{\text {corr }}\left[\mathrm{CCSD}\left(\mathrm{F} 12^{*}\right)\right] / E_{\text {corr }}[\mathrm{CCSD}]$ ratio instead.

Regarding the accuracy of wavefunction $a b$ initio methods, the several flavors of MP2 and SCS-MP2 methods have again shown that they can be parametrized for a specific kind of interaction, but at the cost of degrading the other interactions. While MP3 overcorrects, MP2.5, averaging the MP2 and MP3 values, yields excellent performance. Uncorrected CCSD yields no adequate return for the additional computational effort: similar to dRPA75, however, adding in a D2 correction to compensate for the missing ( $\mathrm{T}$ ) results in an excellent RMSD $=0.16 \mathrm{kcal} \mathrm{mol}^{-1}$ (for $s_{6}=0.228$ ); even further improvement at zero added cost is possible through SCS(MI)CCSD, or even simple overall scaling of the CCSD correlation energy.

Finally, with the relatively small cc-pVDZ-F12 basis set, $\operatorname{CCSD}\left(\mathrm{F} 12^{*}\right)$ has a small but significant edge over CCSD-F12b, particularly for multiply $\mathrm{H}$-bonded systems.

\section{Acknowledgements}

MKK acknowledges a Feinberg Graduate School postdoctoral fellowship. This research was supported by the Israel Science Foundation (grant 1358/15), the Minerva Foundation, the Lise Meitner-Minerva Center for Computational Quantum Chemistry, and a grant from the Yeda-Sela Initiative (Weizmann Institute of Science). The authors would like to thanks Profs. Leeor Kronik (Weizmann Institute of Science), Amir Karton (U. of Western Australia, Perth), and Martin Suhm (U. of Göttingen, Germany) for helpful discussions.

\section{References}

1 K. E. Riley and P. Hobza, Wiley Interdiscip. Rev.: Comput. Mol. Sci., 2011, 1, 3-17.

2 K. E. Riley and P. Hobza, Acc. Chem. Res., 2013, 46, 927-936.

3 M. J. Robertson, J. Tirado-Rives and W. L. Jorgensen, J. Chem. Theory Comput., 2015, 11, 3499-3509.

4 J. J. P. Stewart, J. Mol. Model., 2013, 19, 1-32.

5 B. P. Martin, C. J. Brandon, J. J. P. Stewart and S. B. BraunSand, Proteins: Struct., Funct., Bioinf., 2015, 83, 1427-1435.

6 G. Jansen, Wiley Interdiscip. Rev.: Comput. Mol. Sci., 2014, 4, 127-144.

7 E. G. Hohenstein and C. D. Sherrill, Wiley Interdiscip. Rev.: Comput. Mol. Sci., 2012, 2, 304-326.

8 K. Szalewicz, Wiley Interdiscip. Rev.: Comput. Mol. Sci., 2012, 2, 254-272.

9 J. Rezáč, K. E. Riley and P. Hobza, J. Chem. Theory Comput., 2011, 7, 2427-2438.

10 I. G. Kaplan, Intermolecular Interactions, John Wiley \& Sons, Ltd, Chichester, UK, 2006.

11 P. Jurecka, J. Sponer, J. Cerný and P. Hobza, Phys. Chem. Chem. Phys., 2006, 8, 1985-1993.

12 M. S. Marshall, L. A. Burns and C. D. Sherrill, J. Chem. Phys., 2011, 135, 194102.

13 J. Řezáč, K. E. Riley and P. Hobza, J. Chem. Theory Comput., 2011, 7, 3466-3470.

14 J. Řezáč, P. Jurečka, K. E. Riley, J. Černý, H. Valdes, K. Pluháčková, K. Berka, T. Řezáč, M. Pitoňák, J. Vondrášek and P. Hobza, Collect. Czech. Chem. Commun., 2008, 73, 1261-1270, see also: http:/www.begdb.com.

15 J. Aragó, E. Ortí and J. C. Sancho-García, J. Chem. Theory Comput., 2013, 9, 3437-3443.

16 F. Yu, J. Chem. Theory Comput., 2014, 10, 4400-4407. 
17 L. Goerigk, H. Kruse and S. Grimme, ChemPhysChem, 2011, 12, 3421-3433.

18 K. E. Riley, J. A. Platts, J. Řezáč, P. Hobza and J. G. Hill, J. Phys. Chem. A, 2012, 116, 4159-4169.

19 J. A. Platts, J. G. Hill, K. E. Riley, J. Řezáč and P. Hobza, J. Chem. Theory Comput., 2013, 9, 330-337.

20 M. J. Turner, S. Grabowsky, D. Jayatilaka and M. A. Spackman, J. Phys. Chem. Lett., 2014, 4249-4255.

21 L. Kong, F. A. Bischoff and E. F. Valeev, Chem. Rev., 2012, 112, 75-107.

22 C. Hättig, W. Klopper, A. Köhn and D. P. Tew, Chem. Rev., 2012, 112, 4-74.

23 S. Ten-no, Chem. Phys. Lett., 2004, 398, 56-61.

24 K. A. Peterson, D. A. Dixon and H. Stoll, J. Phys. Chem. A, 2012, 116, 9777-9782.

25 J. M. L. Martin and M. K. Kesharwani, J. Chem. Theory Comput., 2014, 10, 2085-2090.

26 B. Brauer, M. K. Kesharwani and J. M. L. Martin, J. Chem. Theory Comput., 2014, 10, 3791-3799.

27 K. A. Peterson, M. K. Kesharwani and J. M. L. Martin, Mol. Phys., 2015, 113, 1551-1558.

28 H.-J. Werner, P. J. Knowles, G. Knizia, F. R. Manby, M. Schütz, P. Celani, T. Korona, R. Lindh, A. Mitrushenkov, G. Rauhut, K. R. Shamasundar, T. B. Adler, R. D. Amos, A. Bernhardsson, A. Berning, D. L. Cooper, M. J. O. Deegan, A. J. Dobbyn, F. Eckert, E. Goll, C. Hampel, A. Hesselman, G. Hetzer, T. Hrenar, G. Jansen, C. Köppl, Y. Liu, A. W. Lloyd, R. A. Mata, A. J. May, S. J. McNicholas, W. Meyer, M. E. Mura, A. Nicklass, D. P. O’Neill, P. Palmieri, D. Peng, K. Pflüger, R. M. Pitzer, M. Reiher, T. Shiozaki, H. Stoll, A. J. Stone, R. Tarroni, T. Thorsteinsson and M. Wang, MOLPRO, Version 2012.1, a Package of Ab Initio Programs, University of Cardiff Chemistry Consultants (UC3), Cardiff, Wales, UK, 2012, see also: https://www.molpro.net.

29 F. Furche, R. Ahlrichs, C. Hättig, W. Klopper, M. Sierka and F. Weigend, Wiley Interdiscip. Rev.: Comput. Mol. Sci., 2013, 4, 91-100, see also: http://www.turbomole.com.

30 M. J. Frisch, G. W. Trucks, H. B. Schlegel, G. E. Scuseria, M. A. Robb, J. R. Cheeseman, G. Scalmani, V. Barone, B. Mennucci, G. A. Petersson, H. Nakatsuji, M. Caricato, X. Li, H. P. Hratchian, A. F. Izmaylov, J. Bloino, G. Zheng, J. L. Sonnenberg, M. Hada, M. Ehara, K. Toyota, R. Fukuda, J. Hasegawa, M. Ishida, T. Nakajima, Y. Honda, O. Kitao, H. Nakai, T. Vreven, J. A. Montgomery, Jr., J. E. Peralta, F. Ogliaro, M. Bearpark, J. J. Heyd, E. Brothers, K. N. Kudin, V. N. Staroverov, R. Kobayashi, J. Normand, K. Raghavachari, A. P. Rendell, J. C. Burant, S. S. Iyengar, J. Tomasi, M. Cossi, N. Rega, M. J. Millam, M. Klene, J. E. Knox, J. B. Cross, V. Bakken, C. Adamo, J. Jaramillo, R. Gomperts, R. E. Stratmann, O. Yazyev, A. J. Austin, R. Cammi, C. Pomelli, J. W. Ochterski, R. L. Martin, K. Morokuma, V. G. Zakrzewski, G. A. Voth, P. Salvador, J. J. Dannenberg, S. Dapprich, A. D. Daniels, Ö. Farkas, J. B. Foresman, J. V. Ortiz, J. Cioslowski and D. J. Fox, Gaussian 09 Rev. D01., Gaussian, Inc., Wallingford, CT, 2012, see also: http://www.gaussian.com.
31 F. Neese, Wiley Interdiscip. Rev.: Comput. Mol. Sci., 2012, 2, 73-78, see also: https://orcaforum.cec.mpg.de.

32 F. Weigend and M. Häser, Theor. Chem. Acc., 1997, 97, 331-340.

33 R. A. Kendall and H. A. Früchtl, Theor. Chem. Acc., 1997, 97, 158-163.

34 T. H. Dunning, J. Chem. Phys., 1989, 90, 1007.

35 R. A. Kendall, T. H. Dunning and R. J. Harrison, J. Chem. Phys., 1992, 96, 6796.

36 D. E. Woon and T. H. Dunning, J. Chem. Phys., 1993, 98, 1358.

37 K. A. Peterson, D. E. Woon and T. H. Dunning, J. Chem. Phys., 1994, 100, 7410.

38 J. E. Del Bene, J. Phys. Chem., 1993, 97, 107-110.

39 T. M. Parker, L. A. Burns, R. M. Parrish, A. G. Ryno and C. D. Sherrill, J. Chem. Phys., 2014, 140, 094106.

40 E. Papajak, J. Zheng, X. Xu, H. R. Leverentz and D. G. Truhlar, J. Chem. Theory Comput., 2011, 7, 3027-3034.

41 F. Weigend and R. Ahlrichs, Phys. Chem. Chem. Phys., 2005, 7, 3297.

42 D. Rappoport and F. Furche, J. Chem. Phys., 2010, 133, 134105.

43 F. Weigend, J. Comput. Chem., 2008, 29, 167-175.

44 C. Hättig, Phys. Chem. Chem. Phys., 2005, 7, 59.

45 K. A. Peterson, T. B. Adler and H.-J. Werner, J. Chem. Phys., 2008, 128, 084102.

46 K. E. Yousaf and K. A. Peterson, J. Chem. Phys., 2008, 129, 184108.

47 K. E. Yousaf and K. A. Peterson, Chem. Phys. Lett., 2009, 476, 303-307.

48 J. G. Hill, K. A. Peterson, G. Knizia and H.-J. Werner, J. Chem. Phys., 2009, 131, 194105.

49 T. B. Adler, G. Knizia and H.-J. Werner, J. Chem. Phys., 2007, 127, 221106.

50 J. Noga and J. Šimunek, Chem. Phys., 2009, 356, 1-6.

51 O. Marchetti and H.-J. Werner, Phys. Chem. Chem. Phys., 2008, 10, 3400.

52 O. Marchetti and H.-J. Werner, J. Phys. Chem. A, 2009, 113, 11580-11585.

53 S. Grimme, J. Chem. Phys., 2003, 118, 9095.

54 A. Szabados, J. Chem. Phys., 2006, 125, 214105.

55 S. Grimme, L. Goerigk and R. F. Fink, Wiley Interdiscip. Rev.: Comput. Mol. Sci., 2012, 2, 886-906.

56 R. F. Fink, J. Chem. Phys., 2010, 133, 174113.

57 J. P. Perdew and K. Schmidt, AIP Conf. Proc., 2001, 577, 1-20.

58 A. D. Becke, Phys. Rev. A: At., Mol., Opt. Phys., 1988, 38, 3098-3100.

59 J. P. Perdew, Phys. Rev. B: Condens. Matter Mater. Phys., 1986, 33, 8822-8824.

60 C. Lee, W. Yang and R. G. Parr, Phys. Rev. B: Condens. Matter Mater. Phys., 1988, 37, 785-789.

61 J. P. Perdew, K. Burke and M. Ernzerhof, Phys. Rev. Lett., 1996, 77, 3865-3868.

62 J. Tao, J. Perdew, V. Staroverov and G. Scuseria, Phys. Rev. Lett., 2003, 91, 146401.

63 Y. Zhao and D. G. Truhlar, Theor. Chem. Acc., 2008, 120, 215-241. 
64 A. D. Becke, J. Chem. Phys., 1993, 98, 1372.

65 A. D. Becke, J. Chem. Phys., 1993, 98, 5648.

66 P. J. Stephens, F. J. Devlin, C. F. Chabalowski and M. J. Frisch, J. Phys. Chem., 1994, 98, 11623-11627.

67 Y. Wang and J. P. Perdew, Phys. Rev. B: Condens. Matter Mater. Phys., 1991, 44, 13298-13307.

68 C. Adamo and V. Barone, J. Chem. Phys., 1999, 110, 6158.

69 S. Grimme, J. Phys. Chem. A, 2005, 109, 3067-3077.

70 M. M. Quintal, A. Karton, M. A. Iron, A. D. Boese and J. M. L. Martin, J. Phys. Chem. A, 2006, 110, 709-716.

71 A. Austin, G. A. Petersson, M. J. Frisch, F. J. Dobek, G. Scalmani and K. Throssell, J. Chem. Theory Comput., 2012, 8, 4989-5007.

72 R. Peverati and D. G. Truhlar, J. Phys. Chem. Lett., 2011, 2, 2810-2817.

73 T. Yanai, D. P. Tew and N. C. Handy, Chem. Phys. Lett., 2004, 393, 51-57.

74 O. A. Vydrov and G. E. Scuseria, J. Chem. Phys., 2006, 125, 234109.

75 Y.-S. Lin, G.-D. Li, S.-P. Mao and J.-D. Chai, J. Chem. Theory Comput., 2013, 9, 263-272.

76 N. Mardirossian and M. Head-Gordon, Phys. Chem. Chem. Phys., 2014, 16, 9904-9924.

77 S. Grimme, J. Chem. Phys., 2006, 124, 034108.

78 A. Karton, A. Tarnopolsky, J.-F. Lamère, G. C. Schatz and J. M. L. Martin, J. Phys. Chem. A, 2008, 112, 12868-12886.

79 S. Kozuch and J. M. L. Martin, Phys. Chem. Chem. Phys., 2011, 13, 20104-20107.

80 S. Kozuch and J. M. L. Martin, J. Comput. Chem., 2013, 34, 2327-2344.

81 P. D. Mezei, G. I. Csonka, A. Ruzsinszky and M. Kállay, J. Chem. Theory Comput., 2015, 11, 4615-4626.

82 H. Eshuis, J. E. Bates and F. Furche, Theor. Chem. Acc., 2012, 131, 1084.

83 J. Klimeš and A. Michaelides, J. Chem. Phys., 2012, 137, 120901.

84 S. Grimme, J. Comput. Chem., 2006, 27, 1787-1799.

85 T. Schwabe and S. Grimme, Phys. Chem. Chem. Phys., 2007, 9, 3397-3406.

86 S. Grimme, J. Antony, S. Ehrlich and H. Krieg, J. Chem. Phys., 2010, 132, 154104.

87 S. Grimme, S. Ehrlich and L. Goerigk, J. Comput. Chem., 2011, 32, 1456-1465.

88 M. J. D. Powell, The BOBYQA algorithm for bound constrained optimization without derivatives (DAMPT Report 2009/NA06), Department of Applied Mathematics and Theoretical Physics, University of Cambridge, UK, 2009, see also: http://en.wikipe dia.org/wiki/BOBYQA [Retrieved February 29, 2016].

89 O. A. Vydrov and T. Van Voorhis, J. Chem. Phys., 2010, 133, 244103.
90 W. Hujo and S. Grimme, J. Chem. Theory Comput., 2011, 7, 3866-3871.

91 M. K. Kesharwani, A. Karton and J. M. L. Martin, J. Chem. Theory Comput., 2016, 12, 444-454.

92 J. M. Turney, A. C. Simmonett, R. M. Parrish, E. G. Hohenstein, F. A. Evangelista, J. T. Fermann, B. J. Mintz, L. A. Burns, J. J. Wilke, M. L. Abrams, N. J. Russ, M. L. Leininger, C. L. Janssen, E. T. Seidl, W. D. Allen, H. F. Schaefer, R. A. King, E. F. Valeev, C. D. Sherrill and T. D. Crawford, Wiley Interdiscip. Rev.: Comput. Mol. Sci., 2012, 2, 556-565.

93 A. Karton and J. M. L. Martin, Theor. Chem. Acc., 2005, 115, 330-333.

94 D. W. Schwenke, J. Chem. Phys., 2005, 122, 14107.

95 D. S. Ranasinghe and G. A. Petersson, J. Chem. Phys., 2013, 138, 144104.

96 C. Hättig, D. P. Tew and A. Köhn, J. Chem. Phys., 2010, 132, 231102.

97 T. Takatani, E. G. Hohenstein, M. Malagoli, M. S. Marshall and C. D. Sherrill, J. Chem. Phys., 2010, 132, 144104.

98 A. Halkier, H. Koch, P. Jørgensen, O. Christiansen, I. M. B. Nielsen and T. Helgaker, Theor. Chem. Acc., 1997, 97, 150-157.

99 L. A. Burns, M. S. Marshall and C. D. Sherrill, J. Chem. Theory Comput., 2014, 10, 49-57.

100 A. Hesselmann, J. Chem. Phys., 2008, 128, 144112.

101 R. A. DiStasio, G. von Helden, R. P. Steele and M. HeadGordon, Chem. Phys. Lett., 2007, 437, 277-283.

102 G. Chałasiński and M. M. Szcześniak, Mol. Phys., 1988, 63, 205-224.

103 A. Szabo and N. S. Ostlund, J. Chem. Phys., 1977, 67, 4351.

104 R. Sedlak, K. E. Riley, J. Rezáč, M. Pitoňák and P. Hobza, ChemPhysChem, 2013, 14, 698-707.

105 M. Pitoňák and A. Heßelmann, J. Chem. Theory Comput., 2010, 6, 168-178.

106 J. Řezáč, K. E. Riley and P. Hobza, J. Chem. Theory Comput., 2014, 10, 1359-1360.

107 J. Toulouse, W. Zhu, A. Savin, G. Jansen and J. G. Ángyán, J. Chem. Phys., 2011, 135, 084119.

108 G. E. Scuseria, T. M. Henderson and D. C. Sorensen, J. Chem. Phys., 2008, 129, 231101.

109 D. Gruzman, A. Karton and J. M. L. Martin, J. Phys. Chem. A, 2009, 113, 11974-11983.

110 J. M. L. Martin, J. Phys. Chem. A, 2013, 117, 3118-3132.

111 M. K. Kesharwani, B. Brauer and J. M. L. Martin, J. Phys. Chem. A, 2015, 119, 1701-1714.

112 R. M. Parrish, T. M. Parker and C. D. Sherrill, J. Chem. Theory Comput., 2014, 10, 4417-4431. 